\title{
CONVERGENCE OF ITERATES OF A TRANSFER OPERATOR, APPLICATION TO DYNAMICAL SYSTEMS AND TO MARKOV CHAINS
}

\author{
Jean-Pierre Conze $^{1}$ And Albert Raugi ${ }^{1}$
}

\begin{abstract}
We present a spectral theory for a class of operators satisfying a weak "Doeblin-Fortet" condition and apply it to a class of transition operators. This gives the convergence of the series $\sum_{k \geq 0} k^{r} P^{k} f, r \in \mathbb{N}$, under some regularity assumptions and implies the central limit theorem with a rate in $n^{-\frac{1}{2}}$ for the corresponding Markov chain. An application to a non uniformly hyperbolic transformation on the interval is also given.
\end{abstract}

Mathematics Subject Classification. 60J10, 37A05, 37A25.

Received January 28, 2002.

\section{INTRODUCTION}

Given a metric compact space $E$, we consider operators $P$ of the following form:

$$
\operatorname{Pf}(x)=\sum_{s \in S} u_{s}(x) f(s x)
$$

where $\left\{u_{s}, s \in S\right\}$ is a family of non negative functions satisfying a condition of regularity and $\{x \rightarrow s x, s \in S\}$ a family of contracting applications of $E$ into itself.

These operators arise in the theory of Markov chains as transition operators and in ergodic theory as transfer operators associated to Gibbs measures. They are related to dynamical systems of hyperbolic type through the coding given by Markovian partitions [2,24] (see [1] for a general reference for transfer operators and decay of correlations).

In the Markovian case, when the functions $u_{s}$ are Hölderian, the spectral theory of quasi-compact operators is the main tool in the study of the asymptotic behavior of the corresponding Markov chains (cf. [11]). For less regular functions $\left\{u_{s}: s \in S\right\}$, cones methods $[15,16]$ can be applied (see also $[19,26]$ ). We follow here a method introduced in [21] and [6], in which the weights $u_{s}$ are not supposed to be strictly positive.

Under weaker regularity assumptions on the functions $u_{s}$, we have established in [6] results on the convergence of the potential series, with applications to the central limit theorem, the rate of mixing and a Borel-Cantelli type property for the corresponding Gibbs measures.

In the present paper, we give several improvements of these results. In Section 1 a general spectral theory for a class of operators satisfying a weak "Doeblin-Fortet" condition is presented. In Section 2 we establish the

\footnotetext{
Keywords and phrases: Transfer operator, convergence of iterates, Markov chains, rate in the TCL for dynamical systems, Borel-Cantelli property, non uniformly hyperbolic map.

${ }^{1}$ IRMAR, Université de Rennes I, Campus de Beaulieu, 35042 Rennes Cedex, France; e-mail: conze@univ-rennes1.fr
} 
inequalities for a class of transfer operators $P$ of the form $\left(^{*}\right)$. In Sections 3 and 4 we apply these results to get the convergence of the series $\sum_{k>0} k^{r} P^{k} f, r \in \mathbb{N}$, under some regularity assumptions. Using a result of Rio, this gives in Section 5 the optimal rate in the CLT for the Markov chains associated to these operators, under weak regularity assumptions. An other application, the Borel-Cantelli property for a class of dynamical systems, is also considered in Section 6. In the last section we consider an example of a non uniformly hyperbolic system close to models which have been studied by several authors $[12,17,20]$ and show how our method can be applied.

\section{Spectral theory under a Weak Doeblin-Fortet COndition}

In this section, we consider an operator $P$ acting on a normed $\mathbb{C}$-linear space $(B,\|\|)$. We assume that $P$ is power bounded, i.e. such that:

$$
\sup _{n \geq 0,\|f\| \leq 1}\left\|P^{n} f\right\|=M<\infty .
$$

We denote by $\mathcal{K}$ the set of complex numbers of modulus 1 and by $*$ the convolution on the space of sequences defined on $\mathbb{Z}$ with support in $\mathbb{Z}^{+}$.

\subsection{Hypotheses and notations}

We suppose that there exists a sequence of semi-norms $\left(||_{k}\right)_{k \geq 0}$ on $B$ such that:

$\left.H_{1}\right)$ the ball $\left\{f \in B:|f|_{0}+\|f\| \leq 1\right\}$ is relatively compact in $(B,\|\|)$;

$H_{2}$ ) for every $f \in B, \lim _{k \rightarrow+\infty}|f|_{k}=0$;

$H_{3}$ ) there exist a real $C>0$ and a positive convergent series $\sum_{k \geq 0} a_{k}$ such that

$$
\forall f \in B, \forall n \geq 1,\left|P^{n} f\right|_{0} \leq C|f|_{n}+\sum_{j=0}^{n-1} a_{n-1-j}\left\|P^{j} f\right\| .
$$

We set $\rho=\sum_{n \geq 0} a_{n}$ and, for $f \in B, N(f)=\|f\|+\sum_{n \geq 0}|f|_{n}$. The semi-norm | | 0 will be simply denoted by || .

Remark that $H_{3}$ is satisfied (with $C=1$ ) if there exists a positive convergent series $\sum_{k \geq 0} a_{k}$ such that

$$
\forall f \in B, \forall k \geq 0,|P f|_{k} \leq|f|_{k+1}+a_{k}\|f\|
$$

since this condition implies the inequalities:

$$
\left|P^{n} f\right|_{k} \leq|f|_{k+n}+\sum_{j=0}^{n-1} a_{n-1-j+k}\left\|P^{j} f\right\|, \forall n \geq 1, k \geq 0 .
$$

Remark also that we can suppose that the sequence of semi-norms $(||)_{n \geq 0}$ is decreasing, replacing if necessary $|f|_{n}$ with $\sup \left\{|f|_{p}, p \geq n\right\}$ for $n \geq 0$.

In the following, we denote by $W_{1}$ the subspace of $B$ generated by the eigenvectors of $P$ corresponding to eigenvalues of modulus 1 and by $W_{2}$ the subspace $W_{2}=\left\{f \in B: \lim _{n \rightarrow+\infty}\left\|P^{n} f\right\|=0\right\}$.

Theorem 1.1. Under the hypotheses (1.1) we have:

1) the subspace $W_{1}$ is finite dimensional and $B=W_{1} \oplus W_{2}$. If $\lambda$ is a complex number of modulus 1 and $f \in B$, the sequence $\left(\frac{1}{n} \sum_{k=0}^{n-1} \lambda^{-k} P^{k} f\right)_{n \geq 1}$ converges in norm \|\| either to zero or to a $\lambda$-eigenvector of $P$; 
2) for every $\varepsilon>0$, there exists an integer $q=q(\varepsilon) \geq 0$ such that, for every $f$ in $W_{2}$ and every integer $n \geq 0$,

$$
\left|P^{n} f\right|+\varepsilon\left\|P^{n} f\right\| \leq \sum_{p \geq 0}\left(\gamma_{q}^{* p} * \beta_{q, f}\right)(n) ;
$$

where $\gamma_{q}$ and $\beta_{q, f}$ are the sequences defined by:

$$
\begin{aligned}
& \gamma_{q}(n)=\frac{1}{2(\rho+\varepsilon M)} \begin{cases}a_{n-q-1}, & \text { if } n \geq q+2 \\
a_{0}+\varepsilon M, & \text { if } n=q+1 \\
0, & \text { if } n \leq q\end{cases} \\
& \text { and } \beta_{q, f}(n)= \begin{cases}C|f|_{n}+M \sum_{j=n-q+1}^{n} a_{j-1}\|f\|, & \text { if } n \geq q \\
C|f|_{n}+M \sum_{j=1}^{n} a_{j-1}\|f\|, & \text { if } 1 \leq n \leq q-1 \\
|f|, & \text { if } n=0 \\
0, & \text { if } n \leq 0 .\end{cases}
\end{aligned}
$$

If $f$ is in $W_{2}$, we have $\lim _{n}\left|P^{n} f\right|=0$;

3) for every integer $r \geq 0$, there are positive constants $A_{r}$ and $B_{r, q}$ such that, for every $f \in W_{2}$,

$$
\sum_{n \geq 0} n^{r}\left(\left|P^{n} f\right|+\varepsilon\left\|P^{n} f\right\|\right) \leq A_{r} \sum_{n \geq 0} n^{r}|f|_{n}+B_{r, q} N(f) \sum_{n \geq 0} n^{r} a_{n} .
$$

When there exists a real $A>0$ such that $\|f\| \leq A|f|, \forall f \in W_{2}$, we can take $\varepsilon=0$ in the previous assertions.

\subsection{Remark}

Power bounded operators satisfying $H_{1}, H_{2}, H_{3}$ are not in general quasi-compact and do not satisfy the "spectral gap property", but they are close to the following class of quasi-compact operators $P$ which has been considered by Ionescu-Tulcea and Marinescu (cf. for example [11]).

Let $P$ be a power bounded operator acting on a normed linear space $(B,\|\|)$ and let | | be a norm on $B$ such that:

i) the unit ball $\{f \in B:|f| \leq 1\}$ is relatively compact in $(B,\|\|)$;

ii) there exist $\theta \in] 0,1[$ and $c>0$ such that,

$$
|P f| \leq \theta|f|+c\|f\|, \forall f \in B) .
$$

Let us show how to get for this class of operators the spectral gap from Theorem 1.1. Considering on $B$ the decreasing sequence of norms defined by $|f|_{n}=\theta^{n}|f|, n \geq 0$, we have, for every $f \in B$ and every integer $n \geq 0$,

$$
|P f|_{n} \leq|f|_{n+1}+c \theta^{n}\|f\| .
$$

In that case, the sequences $\gamma_{q(0)}$ and $\beta_{q(0), f}$ are bounded respectively by the sequences:

$$
\gamma(n)= \begin{cases}\frac{1-\theta}{2} \theta^{n-q-1}, & \text { if } n \geq q+1 \\ 0, & \text { if } n \leq q\end{cases}
$$




$$
\text { and } \beta_{f}(n)= \begin{cases}B_{f} \theta^{n}, & \text { if } n \geq 0 \\ 0, & \text { if } n<0,\end{cases}
$$

for some strictly positive real $B_{f}$.

For every integers $p \geq 0$ and every $t>0$ such that $\theta t<1$, we have:

$$
\begin{aligned}
\sum_{p \geq 0} \sum_{n \geq 0}\left(\gamma^{* p} * \beta\right)(n) t^{n} & =\sum_{p \geq 0}\left(\sum_{n \geq 0} \gamma(n) t^{n}\right)^{p}\left(\sum_{n \geq 0} \beta(n) t^{n}\right) \\
& =\sum_{p \geq 0} B_{f}\left(\frac{1-\theta}{2}\right)^{p} t^{p(q+1)}\left(\frac{1}{1-\theta t}\right)^{p+1} \\
& =\frac{B_{f}}{1-\theta t-\frac{1-\theta}{2} t^{q+1}} .
\end{aligned}
$$

Consider the polynomial $R(t)=1-\theta t-\left(\frac{1-\theta}{2}\right) t^{q+1}$. For $q=0$ the root of $R$ is $t_{0}=\frac{2}{1+\theta}$. For $q \geq 1$ the polynomial $R$ admits a unique positive real root $t_{0}$, which is simple and strictly between 1 and $1+\frac{1}{q+1}$ and the other roots have a modulus $>t_{0}$. Therefore there exists a real $C_{f}>0$ such that

$$
\sum_{p \geq 0}\left(\gamma^{* p} * \beta\right)(n) \leq C_{f} t_{0}^{-n}, \forall n \in \mathbb{N} .
$$

We can apply Theorem 1.1: if $f$ is in the corresponding subspace $W_{2}$, we have that $\left|P^{n} f\right|$ is bounded by $C_{f} t_{0}^{-n}$.

Example. Explicit examples of applications are given in Sections 6 and 7. We give here a simple example to illustrate Theorem 1.1 and the Remark. We consider the transformation defined on $\mathbb{T}$ by $x \rightarrow 2 x \bmod 1$. The dual operator (for the Lebesgue measure) is $P$ defined by $\operatorname{Pf}(x)=\frac{1}{2}\left(f\left(\frac{x}{2}\right)+f\left(\frac{x+1}{2}\right)\right), f \in L^{2}(\mathbb{T})$.

Let $\Phi: \mathbb{R}_{+} \rightarrow \mathbb{R}_{+}$be a strictly increasing continuous function such that $\Phi(0) \stackrel{2}{=} 0$. For $f \in C(\mathbb{T})$, we set:

$$
\begin{aligned}
m_{\Phi}(f) & =\sup _{x \neq y} \frac{|f(x)-f(y)|}{\Phi(d(x, y))}, \\
\|f\| & =\sup _{x \in \mathbb{T}}|f(x)|,|f|_{\Phi}=\|f\|+m_{\Phi}(f) .
\end{aligned}
$$

Let $B_{\Phi}=\left\{f \in C(\mathbb{T}):|f|_{\Phi}<+\infty\right\}$. For $\Phi(x)=x^{\alpha}, 0<\alpha \leq 1$, the operator $P$ acting on the triple $\left(B_{\Phi},\|\|,||_{\Phi}\right)$ satisfies (2) with $\theta=2^{-\alpha}$. On the other hand, if we take $\Phi(x)=\frac{1}{1+|\ln x|^{\alpha}}, \alpha>0$, then (2) is not satisfied, but we can apply Theorem 1.1.

\subsection{Proof of Theorem $\mathbf{1 . 1}$}

Proof. The proof is given in several steps.

Step 1a. Let $h$ be a non zero vector in $W_{1}$. There exist an integer $p \geq 1$ and, for $1 \leq j \leq p$, complex numbers $z_{j}$ and eigenvectors $f_{j}$ for $P$ corresponding to eigenvalues $\lambda_{j}$ in $\mathcal{K}$ such that $h=\sum_{j=1}^{p} z_{j} f_{j}$.

From $H_{3}$, it results $\left|\sum_{j=1}^{p} z_{j} \lambda_{j}^{n} f_{j}\right| \leq C|h|_{n}+\rho M\|h\|, \forall n \geq 1$.

Taking a strictly increasing sequence of integers $(\varphi(n))_{n \geq 0}$ for which the sequences $\left(\lambda_{j}^{\varphi(n)}\right)_{n \geq 0}, 1 \leq j \leq p$, converge simultaneously to 1 , we get, taking into account the condition $H_{2},|h| \leq \rho M\|h\|$.

$H_{1}$ implies that the set $\left\{\frac{h}{\|h\|}: h \in W_{1}, h \neq 0\right\}$ is relatively compact in $(B,\|\|)$ and therefore the subspace $W_{1}$ is finite dimensional. 
Step 1b. Let us show that, for all $\lambda$ in $\mathcal{K}$ and all $f$ in $B$, the sequence $\left(S_{n, \lambda} f=\frac{1}{n} \sum_{k=0}^{n-1} \lambda^{-k} P^{k} f\right)_{n \geq 1}$ converges in $(B,\|\|)$ either to zero or to a $\lambda$-eigenvector $\Pi_{\lambda}(f)$ of $P$.

Let $f \in B$. From $H_{3}$, we deduce $\left|P^{n} f\right| \leq C|f|_{n}+\rho M\|f\|, \forall n \geq 1$, and therefore $\left|S_{n, \lambda} f\right| \leq C|f|_{n}+\rho M\|f\|$. From $H_{1}$, it follows that the family $\left\{S_{n, \lambda} f: n \geq 1\right\}$ is relatively compact in $(B,\|\|)$. It is clear that any non null cluster value of the sequence $\left(S_{n, \lambda} f\right)_{n>1}$ is a $\lambda$-eigenvector for $P$.

On an other hand, for every integer $p \geq 1$ and every $\lambda$-eigenvector $h$ of $P$, writing $n=\ell p+r$ (Euclidean division), we get:

$$
S_{n, \lambda} f-h=\frac{1}{n}\left(p \sum_{j=0}^{\ell-1} \lambda^{-j} P^{j}\left(S_{p, \lambda} f-h\right)+r \lambda^{-\ell} P^{\ell}\left(S_{r, \lambda}(f-h)\right)\right)
$$

and therefore

$$
\limsup _{n \rightarrow+\infty}\left\|S_{n, \lambda} f-h\right\| \leq M\left\|S_{p, \lambda} f-h\right\| .
$$

This inequality shows that the sequence $\left(S_{n, \lambda} f\right)_{n>1}$ can have only one cluster value; hence the convergence.

Consider $f \in B$. Since $W_{1}$ is finite dimensional, there are only finitely many complex numbers $\lambda$ in $\mathcal{K}$ for which $\Pi_{\lambda} f$ is non zero. Let $\left\{\lambda_{j}: 1 \leq j \leq p\right\}$ be the set of these complex numbers. The vector $g=f-\sum_{j=1}^{p} \Pi_{\lambda_{j}} f$ satisfies then $\lim _{n \rightarrow+\infty} S_{n, \lambda} g=0$, for all $\lambda$ in $\mathcal{K}$.

Step 1c. We show now that if $g$ is a vector in $B$ such that $\lim _{n \rightarrow+\infty} S_{n, \lambda} g=0$, for all $\lambda$ in $\mathcal{K}$, then $g$ belongs to $W_{2}$. This will complete the proof of the first assertion of Theorem 1.1.

Let $g$ be a vector in $B$ satisfying the previous property. Let $h_{0}$ be a cluster value of the sequence $\left(P^{n} g\right)_{n \geq 0}$. There exists a strictly increasing sequence of integers $(\varphi(n))_{n \geq 0}$ such that $\left(P^{\varphi(n)} g\right)_{n \geq 0}$ converges in $(B,\|\|)$ to $h_{0}$. Using the diagonal process and taking a subsequence still denoted by $(\varphi(n))_{n \geq 0}$, we may assume that, for all integers $k \geq 0$, the sequence $\left(P^{\varphi(n)-k} f\right)_{n>0}$ converges in $(B,\|\|)$ to a vector denoted by $h_{k}$.

The sequence $\left(h_{k}\right)_{k \geq 0}$ satisfies $P h_{k+1}=h_{k}, \forall k \geq 0$. This relation can be extended to $\mathbb{Z}$ by setting, for every $k \geq 1, h_{-k}=P^{k} h_{0}$. It is clear that the family $\left\{h_{k}: k \in \mathbb{Z}\right\}$ is relatively compact in $(B,\|\|)$. For all $\lambda$ in $\mathcal{K}$ and all integers $p \in \mathbb{Z}$, we have in $(B,\|\|) \lim _{n \rightarrow+\infty} \frac{1}{n} \sum_{k=0}^{n-1} \lambda^{k} h_{p-k}=0$.

One shows easily that, for any linear continuous form $\psi$ on $(B,\|\|)$, the sequence $u=\left(\psi\left(h_{-k}\right)\right)_{k \in \mathbb{Z}}$ in $\ell^{\infty}(\mathbb{Z})$ is almost periodic (i.e. the set of sequences $\left\{u_{p}=\left(\psi\left(h_{p-k}\right)\right)_{k \in \mathbb{Z}}: p \in \mathbb{N}\right\}$ is relatively compact in $\left.\ell^{\infty}(\mathbb{Z})\right)$. Moreover, we have $\lim _{n \rightarrow+\infty} \frac{1}{n} \sum_{k=0}^{n-1} \lambda^{k} u_{k}=0, \forall \lambda \in \mathcal{K}$. This implies that $u_{k}=0, \forall k \in \mathbb{Z}$.

We deduce from it that zero is the only cluster value of the sequence $\left(P^{n} g\right)_{n \geq 0}$, which therefore converges to zero.

Step 2. Let $\varepsilon>0$. $H_{1}$ implies that the set $B_{2}=\left\{g \in W_{2}:|g|+\varepsilon\|g\|=1\right\}$ is relatively compact in $(B,\|\|)$. As we have $\lim _{n}\left\|P^{n} g\right\|=0, \forall g \in W_{2}$, and $P$ is power bounded, the convergence is uniform on $B_{2}$. Therefore there exists an integer $q=q(\varepsilon) \geq 0$ such that, for every $f \in W_{2}$,

$$
\left\|P^{q} f\right\| \leq \frac{|f|+\varepsilon\|f\|}{2(\rho+\varepsilon M)} .
$$


For $f \in W_{2}$, we have:

- for $n \geq q+1$,

$$
\left|P^{n} f\right|+\varepsilon\left\|P^{n} f\right\| \leq C|f|_{n}+M \sum_{j=0}^{q-1} a_{n-1-j}\|f\|+\frac{1}{2(\rho+\varepsilon M)} \sum_{j=q}^{n-1} b_{n-1-j}\left(\left|P^{j-q} f\right|+\varepsilon\left\|P^{j-q} f\right\|\right),
$$

with $b_{0}=a_{0}+\varepsilon M$ and $\forall n \geq 1, b_{n}=a_{n}$;

- for $1 \leq n \leq q$,

$$
\left|P^{n} f\right| \leq C|f|_{n}+M \sum_{j=0}^{n-1} a_{n-1-j}\|f\|
$$

Let $\alpha_{f}$ be the sequence defined by

$$
\alpha_{f}(n)= \begin{cases}\left|P^{n} f\right|, & \text { if } n \geq 0 \\ 0, & \text { if } n<0\end{cases}
$$

We have

$$
\alpha_{f}(n) \leq \beta_{q, f}(n)+\left(\gamma_{q} * \alpha_{f}\right)(n), \forall n \in \mathbb{Z},
$$

and therefore, for every integer $\ell \geq 1$,

$$
\alpha_{f}(n) \leq \sum_{p=0}^{\ell-1}\left(\gamma_{q}^{* p} * \beta_{q, f}\right)(n)+\left(\gamma_{q}^{* \ell} * \alpha_{f}\right)(n), \forall n \in \mathbb{Z}
$$

As we have $\left(\gamma_{q}^{* \ell} * \alpha_{f}\right)(n)=0$, for $\ell$ such that $\ell(q+1)>n$, this implies inequality (1) of Theorem 1.1.

Step 3. For simplicity, we denote respectively by $\gamma$ and $\beta$ the sequences $\gamma_{q}$ and $\beta_{q, f}$.

We have $\sum_{n \geq 0} \gamma(n)=\frac{1}{2}$ and $\sum_{n \geq 0}\left(\gamma^{* p} * \beta\right)(n)=2^{-p} \bar{\beta}$, where $\bar{\beta}=\sum_{n \geq 0} \beta(n)$, and therefore

$$
\sum_{n \geq 0}\left(\left|P^{n} f\right|+\varepsilon\left\|P^{n} f\right\|\right) \leq 2 \bar{\beta} \leq 2 \max \{1, C\} \sum_{n \geq 0}|f|_{n}+2 q M\|f\| \sum_{n \geq 0} b_{n} .
$$

Let $p$ be an integer $\geq 1$ and let $X_{1}, \ldots, X_{p}, Y$ be independent integer random variables such that:

$$
\forall k \in \mathbb{N}, \mathbb{P}\left[\left\{X_{1}=k\right\}\right]=\ldots=\mathbb{P}\left[\left\{X_{p}=k\right\}\right]=2 \gamma(k) \text { and } \mathbb{P}[\{Y=k\}]=\beta(k) / \bar{\beta} .
$$

For every integer $r \geq 1$, we have

$$
\begin{aligned}
\bar{\beta}^{-1} 2^{p} \sum_{n \geq 1} n^{r}\left(\gamma^{* p} * \beta\right)(n) & =\mathbb{E}\left[\left(X_{1}+\ldots+X_{p}+Y\right)^{r}\right] \\
& \leq(p+1)^{r-1}\left(p \mathbb{E}\left[X_{1}^{r}\right]+\mathbb{E}\left[Y^{r}\right]\right) \\
& \leq(p+1)^{r-1}\left(2 p \sum_{n \geq 1} n^{r} \gamma(n)+\frac{1}{\bar{\beta}} \sum_{n \geq 1} n^{r} \beta(n)\right)
\end{aligned}
$$

It follows that

$$
\sum_{n \geq 1} n^{r}\left(\left|P^{n} f\right|+\varepsilon\left\|P^{n} f\right\|\right) \leq \sum_{p \geq 1} \frac{p(p+1)^{r-1}}{2^{p-1}} \bar{\beta} \sum_{n \geq 1} n^{r} \gamma(n)+\sum_{p \geq 0} \frac{(p+1)^{r-1}}{2^{p}} \sum_{n \geq 1} n^{r} \beta(n) .
$$

From this, one deduces easily the last assertion. 
Notation. Under the hypotheses (1.1), we denote by $\Pi_{1}$ and $\Pi_{2}$ the projectors from $B$ respectively onto $W_{1}$ and $W_{2}$, and by $Q$ the operator $P \circ \Pi_{2}$.

The following lemma will be useful in Sections 5 and 6 .

Lemma 1.2. Under the hypotheses (1.1), let us assume that $(B,\|\|)$ is a normed algebra and that the seminorms $\left.\right|_{k}$ are decreasing and satisfy the inequalities

$$
|f g|_{k} \leq\left.\|f\||| g\right|_{k}+\left.\|g\||| f\right|_{k}, \forall f, g \in B, \forall k \geq 0 .
$$

Moreover, let us assume satisfied the following condition (which extends to the family of semi-norms $\left(||_{k}\right)$ the inequalities $\mathrm{H}_{3}$ satisfied by the semi-norm ||):

there exist $C>0$ and a sequence of positive convergent series $\left(\sum_{n \geq 0} a_{n}^{(k)}\right)_{k \geq 0}$ such that

$$
\forall f \in B, \forall n \geq 1, \forall k \geq 0,\left|P^{n} f\right|_{k} \leq C|f|_{n+k}+\sum_{j=0}^{n-1} a_{n-1-j}^{(k)}\left\|P^{j} f\right\|
$$

Consider the sequence defined by

$$
\forall n \geq 0, \delta(n)= \begin{cases}\sup _{j \geq 0} a_{j}^{(n)} & \text { if } \forall f \in B,|f|_{n}=\left|\Pi_{2} f\right|_{n} \\ \sum_{j \geq 0} a_{j}^{(n)} & \text { else. }\end{cases}
$$

Let $\zeta=\zeta_{f_{1}, \ldots, f_{m}}$ and $\psi_{q}$ be the sequences defined by

$$
\begin{gathered}
\zeta_{f_{1}, \ldots, f_{m}}(n)=\left\|f_{1}\right\| \sum_{i=2}^{m}(i-1)\left(\prod_{\substack{j=2 \\
j \neq i}}^{m}\left\|f_{j}\right\|\right) N\left(f_{i}\right) \delta(n)+\sum_{i=1}^{m}\left(\prod_{\substack{j=1 \\
j \neq i}}^{m}\left\|f_{j}\right\|\right)\left|f_{i}\right|_{n}, \\
\psi_{q}(n)= \begin{cases}\sum_{j=n-q+1}^{n} a_{j-1} & \text { if } n \geq q \\
\sum_{j=1}^{n} a_{j-1} & \text { if } 1 \leq n \leq q-1 \\
0 & \text { if } n=0 .\end{cases}
\end{gathered}
$$

If $\sum_{n \geq 0} \delta(n)<+\infty$, there exists a real $E_{1}>0$ such that, for all integers $m \geq 2$, for all $m$-uples of vectors $f_{1}, \ldots, f_{m}$ in $B$ and all natural integers $k_{2}, \ldots, k_{m}$,

$$
\left|Q^{n} f_{1} Q^{k_{1}} f_{2} \cdots Q^{k_{m}} f_{m}\right|+\left\|Q^{n} f_{1} Q^{k_{2}} f_{2} \cdots Q^{k_{m}} f_{m}\right\| \leq \sum_{p \geq 0}\left(\gamma_{q(1)}^{* p} * \xi\right)(n), \quad \forall n \geq 0
$$

where $\xi=\xi_{f_{1}, \ldots, f_{m}}$ is the sequence defined by

$$
E_{1}^{-m} \xi(n)=\zeta(n)+\prod_{j=1}^{m}\left\|f_{j}\right\|\left(C \delta(n)+\psi_{q}(n)\right)
$$


Proof. There exists a real $D>\max \{M, C\}$ such that, for every integers $k \geq 1$ and $n \geq 0$ and every functions $f$ in $W_{2}$,

$$
\begin{aligned}
\left|P^{k} f\right|_{n} & \leq C|f|_{n+k}+\sum_{j=0}^{k-1} a_{k-j-1}^{(n)}\left\|P^{j} f\right\| \\
& \leq C|f|_{n}+\delta(n) \sum_{j \geq 0}\left\|P^{j} f\right\| \\
& \leq D\left(|f|_{n}+\delta(n) N(f)\right) .
\end{aligned}
$$

It follows that, for every integers $k \geq 1$ and $n \geq 0$, every functions $f$ and $g$ in $W_{2}$,

$$
\begin{aligned}
\left|f P^{k} g\right|_{n} & =\|f\|\left|P^{k} g\right|_{n}+\left\|P^{k} g\right\||f|_{n} \\
& \leq D\left(\|f\||g|_{n}+\|g\||f|_{n}+\delta(n)\|f\| N(g)\right)
\end{aligned}
$$

and therefore there exists $E_{1}>D$ such that

$$
N\left(f P^{k} g\right) \leq D\left(1+\sum_{n \geq 0} \delta(n)\right)(\|f\| N(g)+\|g\| N(f)) \leq E_{1}(\|f\| N(g)+\|g\| N(f)) .
$$

This implies:

$$
\zeta_{f_{1}, \ldots, f_{m-2}, f_{m-1} Q^{k} f_{m}}(n) \leq E_{1} \zeta_{f_{1}, \ldots, f_{m-1}, f_{m}}(n), \forall k \in \mathbb{N} .
$$

We have then that $\left|f_{1} Q^{k_{2}} f_{2}\right| \leq E_{1} \zeta_{f_{1}, f_{2}}(n)$. Suppose that

$$
\left|f_{1} Q^{k_{2}} f_{2} \ldots Q^{k_{m}} f_{m}\right|_{n} \leq E_{1}^{m-1} \zeta_{f_{1}, \ldots, f_{m}}(n),
$$

then we have

$$
\begin{aligned}
\left|f_{1} Q^{k_{2}} f_{2} \ldots Q^{k_{m}} f_{m} Q^{k_{m+1}} f_{m+1}\right|_{n} & \leq E_{1}^{m-1} \zeta_{f_{1}, \ldots, f_{m-1}, f_{m} Q^{k_{m+1} f_{m+1}}}(n) \\
& \leq E_{1}^{m} \zeta_{f_{1}, \ldots, f_{m-1}, f_{m}, f_{m+1}}(n) .
\end{aligned}
$$

So we have proved recursively (3).

On an other hand, we have:

$$
\begin{aligned}
\left|\Pi_{1} f_{1} Q^{k_{2}} f_{2} \ldots Q^{k_{m}} f_{m}\right|_{n} & \leq M \delta(n)\left\|\Pi_{1} f_{1} Q^{k_{2}} f_{2} \ldots Q^{k_{m}} f_{m}\right\| \\
& \leq M^{m} \delta(n)\left\|f_{1}\right\| \ldots\left\|f_{m}\right\| .
\end{aligned}
$$

The desired result follows from the second assertion of Theorem 1.1 and the inequality

$$
\beta_{q, \Pi_{2} f_{1} Q^{k_{2}} f_{2} \ldots Q^{k_{m}} f_{m}} \leq E_{1}^{m}\left(\zeta(n)+\prod_{j=1}^{m}\left\|f_{j}\right\|\left(C \delta(n)+\psi_{q}(n)\right)\right) .
$$

\section{INEQUALITIES FOR TRANSFER OPERATORS}

We describe now a class of operators to which the results of Section 1 will be applied. 


\subsection{Hypotheses}

Let $(E, d)$ be a compact metric space. We consider a finite or countable family $S$ of continuous applications $s: x \rightarrow s x$ from $E$ into itself. The following contraction hypothesis will be assumed afterwards (except in the last section where a case of non uniform contraction will be discussed):

there exists a sequence of positive real numbers $\left(\eta_{n}\right)_{n \geq 0}$ decreasing to 0 such that:

$$
d(x, y) \leq \eta_{n} \Rightarrow d(s x, s y) \leq \eta_{n+1}, \forall x, y \in E, \forall s \in S
$$

Frequently in the examples, the applications $x \rightarrow s x$ satisfy a uniform condition of contraction: there exists $c<1$ such that $d(s x, s y)<c d(x, y), \forall x, y \in E, x \neq y$. In that case, one can take $\eta_{n}=c^{n} \eta_{0}$, for $n \geq 1$, where $\eta_{0}=\operatorname{diam}(E)$ is the diameter of $E$.

Moreover let be given a family of continuous non-negative functions $\left\{u_{s}: s \in S\right\}$ defined on $E$ such that

$$
\sup _{x \in E} \sum_{s \in S} u_{s}(x)<+\infty
$$

We define a positive kernel $P$ on $E$ by

$$
P f(x)=\sum_{s \in S} u_{s}(x) f(s x)
$$

This kernel acts on the space of bounded functions on $E$, on the cone of positive functions and on the cone $\mathcal{M}^{+}$ of positive measures defined on the Borel $\sigma$-algebra of $E$.

When the family $\left\{u_{s}: s \in S\right\}$ satisfies the condition

$$
\sum_{s \in S} u_{s}(x)=1, \forall x \in E
$$

$P$ is a Markovian operator. We can define a Markov chain with values in $E$ such that, at each step, the transition are possible from a point $y$ to the points $s y, s \in S$, with probability $u_{s}(y)$.

\subsection{Notations}

Let $\mathcal{C}(E)$ be the space of continuous real or complex functions on $E$. For every integer $k \geq 0$ and every function $g \in \mathcal{C}(E)$, we define:

$$
v(g, k)=\sup _{\left\{(x, y) \in E^{2}: d(x, y) \leq \eta_{k}\right\}}|g(x)-g(y)| .
$$

We measure the regularity of the family $\left\{u_{s}, s \in S\right\}$ by the sequence $(w(k, 0))_{k \geq 0}$ defined by:

$$
w(k, 0)=\sup _{\left\{(x, y) \in E^{2}: d(x, y) \leq \eta_{k}\right\}} \sum_{s \in S}\left|u_{s}(x)-u_{s}(y)\right|,
$$

and its "mean regularity" by the following sequences defined for $n \geq 1$ :

$$
w(k, n)=\sup _{\left\{(x, y) \in E^{2}: d(x, y) \leq \eta_{k}\right\}} \sum_{\left(s_{1}, \ldots s_{n}\right) \in S^{n}} u_{s_{n}}\left(s_{n-1} \cdots s_{1} x\right) \ldots u_{s_{1}}(x) \sum_{s \in S}\left|u_{s}\left(s_{n} \cdots s_{1} x\right)-u_{s}\left(s_{n} \cdots s_{1} y\right)\right| .
$$




\subsection{Inequalities}

1) For all natural integers $k, n$, we have:

$$
w(k, n) \leq\left\|P^{n} 1\right\|_{\infty} w(k+n, 0) \leq\left\|P^{n} 1\right\|_{\infty} \sum_{s \in S} v\left(u_{s}, k+n\right) .
$$

Note that, when $P$ is a power bounded operator on the space of bounded functions on $E$ (i.e. $M=\sup _{n \geq 0}$ $\left.\left\|P^{n} 1\right\|_{\infty}<\infty\right)$, we have:

$$
w(k, n) \leq M w(k+n, 0), \forall k \geq 0 .
$$

2) For $a, b>0$, we have: $|a-b|=\left(1-\mathrm{e}^{-\left|\ln \frac{a}{b}\right|}\right) \max \{a, b\} \leq\left|\ln \frac{a}{b}\right|(a+b)$. When the functions $u_{s}$ are strictly positive, we have therefore, for $k, n \in \mathbb{N}$ :

$$
\begin{aligned}
\sum_{s \in S} v\left(u_{s}, n\right) & \leq 2\|P 1\|_{\infty} \sup _{s \in S} v\left(\ln u_{s}, n\right), \\
w(k, n) & \leq 2\left\|P^{n} 1\right\|_{\infty}\|P 1\|_{\infty} \sup _{s \in S} v\left(\ln u_{s}, k+n\right) .
\end{aligned}
$$

3) For any bounded function $f$ on $E$, any integer $n \geq 1$, we have for $x, y$ in $E$ :

$$
\begin{aligned}
P^{n} f(x)-P^{n} f(y)= & \sum_{\left(s_{1}, \ldots, s_{n}\right) \in S^{n}} u_{s_{n}}\left(s_{n-1} \cdots s_{1} x\right) \ldots u_{s_{1}}(x)\left(f\left(s_{n} \cdots s_{1} x\right)-f\left(s_{n} \cdots s_{1} y\right)\right) \\
+ & \sum_{j=2}^{n} \sum_{\left(s_{1}, \ldots, s_{j}\right) \in S^{j}} u_{s_{j-1}}\left(s_{j-2} \cdots s_{1} x\right) \cdots u_{s_{1}}(x) \\
& \left(u_{s_{j}}\left(s_{j-1} \cdots s_{1} x\right)-u_{s_{j}}\left(s_{j-1} \cdots s_{1} y\right)\right) P^{n-j} f\left(s_{j} \cdots s_{1} y\right) \\
+ & \sum_{s_{1} \in S}\left(u_{s_{1}}(x)-u_{s_{1}}(y)\right) P^{n-1} f\left(s_{1} y\right) .
\end{aligned}
$$

From the last equality, we deduce:

Lemma 2.1. If $f$ is a bounded function on $E$ and $k, n$ natural integers, we have:

$$
v\left(P^{n} f, k\right) \leq\left\|P^{n} 1\right\|_{\infty} v(f, k+n)+\sum_{j=0}^{n-1} w(k, n-1-j)\left\|P^{j} f\right\|_{\infty} .
$$

\subsection{An example}

We give now an example to illustrate the different conditions of regularity (see [6] for details). We consider the interval $E=[0,1]$, an integer $q \geq 1$ and a real $\beta$ in $] 0,1[$. Let $t$ be the application from $[0,+\infty[$ into $[0,+\infty[$ defined by:

$$
\forall x \in E, t(x)=\frac{x}{1+q x^{\beta}} .
$$

We have, $\forall x, y \in[0,+\infty[, t(x+y) \leq t(x)+t(y)$ and therefore:

$$
|t(x)-t(y)| \leq t(|x-y|) \leq|x-y| .
$$

The application $t$ sends the interval $[0,1]$ on the interval $\left[0, \frac{1}{1+q}\right]$. 
Let $S$ be the family of continuous transformations of $E$ into itself defined by:

$$
S=\left\{t_{k}: x \rightarrow t(x)+\frac{k}{1+q}: 0 \leq k \leq q\right\} .
$$

From (10), we have:

$$
\left.\forall s_{1}, \ldots, s_{n} \in S,\left|s_{1} \cdots s_{n} x-s_{1} \cdots s_{n} y\right| \leq t^{n}(\mid x-y]\right) \leq t^{n}(1) .
$$

For the sequence $\left(\eta_{k}\right)_{k \geq 0}$ on $E$ defined by $\eta_{k}=t^{k}(1)$, the contraction hypothesis (4) is then satisfied.

Let $\left\{u_{s}: s \in S\right\}$ be a family of functions such that $\sum_{s \in S} u_{s}(x)=1, \forall x \in E$. We assume that there exists a continuous increasing function $\Phi$ from $[0,1]$ to $[0,1]$ such that $\Phi(0)=0$ and

$$
\sum_{s \in S}\left|u_{s}(x)-u_{s}(y)\right| \leq \Phi(|x-y|), \forall x, y \in[0,1] .
$$

We have:

$$
w(n, 0) \leq \Phi\left(\eta_{n}\right), \forall n \in \mathbb{N} .
$$

The sequence $\left(\eta_{n}=t^{n}(1)\right)_{n \geq 0}$ decreases to zero and satisfies $\eta_{n}=(1+o(1))(n q \beta)^{-\frac{1}{\beta}}$.

For $\Phi(x)=x^{\alpha}$, with $0<\alpha \leq 1$, the condition $\sum_{k \geq 0} w(k, 0)<+\infty$ is satisfied if $\frac{\alpha}{\beta}>1$. For $\Phi(x)=\frac{1}{1+|\ln x|^{\alpha}}$, with $\alpha>0$, the condition $\sum_{k \geq 0} w(k, 0)<+\infty$ is not necessarily satisfied.

The convergence of the series $\sum_{n \geq 0} w(0, n)$ can be established under less restrictive conditions:

- for $\Phi(x)=x^{\alpha}$, with $0<\alpha \leq 1$, we have $\sum_{n \geq 0} w(0, n)<+\infty$;

- for $\Phi(x)=\frac{1}{1+|\ln x|^{\alpha}}$, we have $\sum_{n \geq 0} w(0, n)<+\infty$, for $\alpha>1$.

\section{Convergence of $\sum\left\|P^{n} f\right\|_{\infty}$}

\subsection{Hypothesis}

In this section we make the following assumptions:

$$
\sum_{n \geq 0} w(0, n)<+\infty, \lim _{k} \downarrow w(k, 0)=0 .
$$

$$
P \text { is power bounded: } M=\sup _{n \geq 1}\left\|P^{n} 1\right\|_{\infty}<+\infty \text {. }
$$

Remark that, by continuity of the weights $u_{s}$, the second condition in (11) is always satisfied when the family of applications $S$ is finite. When $P$ is Markovian, equation (12) is clearly satisfied. This is true as well, up to a multiplicative constant, when the weights $u_{s}$ are strictly positive (cf. Lem. 3.4).

We will make use of the following elementary lemma:

Lemma 3.1. If $\left(u_{k}\right)_{k \geq 1}$ is a sequence of real numbers decreasing to 0 , there exists a sequence $\left(\varphi_{k}\right)_{k \geq 1}$ such that:

$$
\sum_{k \geq 0} \varphi_{k}=+\infty \quad \text { and } \quad \sum_{k \geq 0} \varphi_{k} u_{k}<+\infty .
$$

Proof. We may take for instance, for some $\alpha \in[1,2[$,

$$
\varphi_{k}=u_{k}^{-\alpha}\left(u_{k}-u_{k+1}\right), k \geq 1 .
$$


From the inequalities $\forall k \in \mathbb{N}, w(k, n) \leq M w(0, n)$, it follows that

$$
\lim _{k \rightarrow+\infty} \downarrow \sum_{n \geq 0} w(k, n)=\sum_{n \geq 0} \lim _{k \rightarrow+\infty} \downarrow w(k, n)=0 .
$$

Consider a function $\varphi$ from $\mathbb{N}$ to $] 0,+\infty[$ such that (cf. Lem. 3.1):

$$
\sum_{k \geq 0} \varphi(k)=+\infty \quad \text { and } \quad \sum_{k \geq 0} \varphi(k) \sum_{n \geq 0} w(k, n)<+\infty .
$$

Notations. For $n, \ell$ non negative integers, we set

$$
\begin{aligned}
a(\ell, n) & =\sum_{k \geq 0} \varphi(k) w(k+\ell, n), \\
\rho(\ell) & =\sum_{n \geq 0} a(\ell, n) .
\end{aligned}
$$

We define semi-norms by setting for a function $f$ on $E$ :

$$
\begin{aligned}
|f|_{\varphi, n} & =\sum_{k \geq 0} \varphi(k) v(f, k+n), n \geq 0, \\
|f|_{\varphi} & =|f|_{\varphi, 0}=\sum_{k \geq 0} \varphi(k) v(f, k) .
\end{aligned}
$$

Let $B_{\varphi}$ be the subspace of $C(E)$ defined by $B_{\varphi}=\left\{f \in C(E):|f|_{\varphi}<\infty\right\}$.

By Lemma 2.1, the semi-norms $\left(||_{\varphi, n}\right)_{n \geq 0}$ on $B_{\varphi}$ satisfy the following inequalities:

$$
\forall n \geq 1, \quad \forall k \geq 0,\left|P^{n} f\right|_{\varphi, k} \leq M|f|_{\varphi, n+k}+\sum_{j=0}^{n-1} a(k, n-j-1)\left\|P^{j} f\right\|_{\infty} .
$$

The inequalities

$$
\forall N \in \mathbb{N},\left(\sum_{k=0}^{N} \varphi(k)\right) v(f, N) \leq \sum_{k=0}^{N} \varphi(k) v(f, k) \leq|f|_{\varphi}
$$

imply that the set of functions $\left\{f \in C(E):|f|_{\varphi}+\|f\|_{\infty} \leq 1\right\}$ is equicontinuous. By Ascoli-Arzela theorem, this set is relatively compact in $\left(C(E),\|\|_{\infty}\right)$. It is easy to see that this set is closed and therefore is a compact subset of $\left(C(E),\|\|_{\infty}\right)$.

It follows that the operator $P$ satisfies the hypotheses of Theorem 1.1 and we have the following theorem, where $W_{1}$ is the subspace of $C(E)$ generated by the eigenvectors of $P$ corresponding to eigenvalues of modulus 1 and $W_{2}=\left\{f \in C(E): \lim _{n \rightarrow+\infty}\left\|P^{n} f\right\|_{\infty}=0\right\}$.

Theorem 3.2. If (3.1) is satisfied, $W_{1}$ is finite dimensional and $C(E)=W_{1} \oplus W_{2}$.

Moreover, for $r \geq 0$, if the condition $\sum_{n \geq 0} n^{r} w(0, n)<+\infty$ is satisfied, we have $\sum_{n \geq 0} n^{r}\left\|P^{n} f\right\|_{\infty}<+\infty$ for all $f \in W_{2}$ such that $\sum_{n \geq 0} n^{r} v(f, n)<+\infty$. 
Proof. If $h \in C(E)$ is an eigenvector of $P$ of modulus 1 , then from (9) we have $\forall k \geq 0, v(h, k) \leq \sum_{j \geq 0} w(k, j)$ $\|h\|_{\infty}$ and therefore $h$ belongs to $B_{\varphi}$ for all $\left.\varphi: \mathbb{N} \rightarrow\right] 0,+\infty[$ such that

$$
\sum_{k \geq 0} \varphi(k)=+\infty \text { and } \sum_{k \geq 0} \varphi(k) \sum_{j \geq 0} w(k, j)<\infty .
$$

Let $f \in C(E)$. As $\lim _{n \rightarrow+\infty} v(f, n)=0$, we can apply Lemma 3.1, with $u(n)=\max \left\{\sum_{j \geq 0} w(n, j), v(f, n)\right\}$, and choose a function $\varphi$ from $\mathbb{N}$ to $] 0,+\infty\left[\right.$ such that $f \in B_{\varphi}$ and the conditions (15) are satisfied.

Then the first assertion results from Theorem 1.1.

Let $f$ such that $\sum_{n \geq 0} n^{r} v(f, n)<+\infty$.

Applying Lemma 3.1 with $u(n)=\max \left\{\sum_{k \geq 0} w(n, k), \sum_{k \geq n} k^{r} v(f, k)\right\}, n \geq 1$, we can choose a function $\varphi$ from $\mathbb{N}$ to $] 0,+\infty[$ such that

$$
\sum_{k \geq 0} \varphi(k)=+\infty ; \sum_{k \geq 0} \varphi(k) \sum_{j \geq 0} w(k, j)<\infty \text { and } \sum_{k \geq 0} \varphi(k) \sum_{j \geq k} j^{r} v(f, j)<\infty .
$$

From Theorem 1.1, we have then

$$
\sum_{n \geq 0} n^{r}\left(\left|P^{n} f\right|_{\varphi}+\left\|P^{n} f\right\|_{\infty}\right)<+\infty
$$

and the result follows.

\subsection{Peripheral spectrum of $P$}

To apply the previous results, we need information on $W_{1}$ and on the peripheral spectrum of $P$. In particular it is important to give conditions which ensure that $\operatorname{dim}\left(W_{1}\right)=1$. We refer to [21] and [6] for a description of the subspace $W_{1}$ of $C(E)$ in terms of ergodic classes for a Markov chain associated to $P$.

The following notion of proximality is useful to give a criterium for $\operatorname{dim}\left(W_{1}\right)=1(c f$. [5]). The family of transitions $x \rightarrow s x$ (allowed if $u_{s}(x)>0$ ), defines a "topological Markov chain" on the space $E$. We denote it by $\left(S,\left(u_{s}\right)_{s \in S}\right)$.

\subsection{Definitions}

We say that a compact set $F$ of $E$ is $p$-invariant (or invariant for $p=1$ ) if, for all $x \in F$ and all $\left(s_{1}, \ldots, s_{p}\right.$ ) $\in S^{p}$ such that $u_{s_{p}}\left(s_{p-1} \cdots s_{1} x\right) \cdots u_{s_{2}}\left(s_{1} x\right) u_{s_{1}}(x)>0$, we have $s_{p} \cdots s_{1} x \in F$.

We say that $\left(S,\left(u_{s}\right)_{s \in S}\right)$ is $p$-proximal if any two $p$-invariant non-empty compact sets intersect. It is strongly proximal if it is $p$-proximal for any integer $p \geq 1$.

Theorem 3.3. (cf. [6,21]): if $P$ is Markovian and if (3.1) is satisfied, we have:

i) the eigenvalues of modulus 1 of $P$ are roots of unity. There exists a finite set $\left\{E_{1}, \ldots, E_{m}\right\}$ of disjoint invariant compact sets (ergodic classes) such that

$$
\left\{\mathbb{P}_{x}\left[\left\{\limsup _{n \rightarrow+\infty} d\left(X_{n}, E_{j}\right)=0\right\}\right] ; 1 \leq j \leq m\right\}
$$

is a basis of the eigenspace of $P$ corresponding to the eigenvalue 1;

ii) each invariant compact set $E_{j}$ supports a unique P-invariant probability measure and splits into a finite number $\left\{C_{j, 1}, \ldots, C_{j, d_{j}}\right\}$ of $d_{j}$-invariant compact sets (cyclic classes); 
iii) the set of functions

$$
\left\{\sum_{\ell=0}^{d_{j}-1} \omega_{j}^{\ell} \mathbb{P}_{x}\left[\left\{\limsup _{n \rightarrow+\infty} d\left(X_{n d_{j}}, C_{j, \ell}\right)=0\right\}\right] ; 1 \leq j \leq m, \omega_{j} \text { is a } d_{j} \text {-root of unity }\right\}
$$

is a basis of $W_{1}$;

iv) if $\left(S,\left(u_{s}\right)_{s \in S}\right)$ is strongly proximal, there exists a unique ergodic class (without cyclic subclasses) supporting the unique $P$-invariant probability measure $\nu$ on $E$ and we have, uniformly on $E$, for all $f \in C(E)$ : $\lim _{n} P^{n} f=\nu(f)$.

\subsection{Existence of an invariant function}

In the general case, we can reduce $P$ (by "relativisation") to a Markovian operator if there exists a strictly positive eigenfunction. The existence of such a function can be shown classically, when the weights $u_{s}$ are $>0$ (cf. $[2,25]$ ), under a regularity condition which slightly stronger then (11) (in the following lemma $P$ is not supposed to be power bounded):

Lemma 3.4. If the functions $u_{s}, s \in S$, are strictly positive and satisfy the condition

$$
\sum_{k \geq 0} \sup _{s \in S} v\left(\ln u_{s}, k\right)<+\infty
$$

the operator $P$ has a strictly positive proper function $h$ satisfying:

$$
v(\ln h, k) \leq \sum_{n \geq k} \sup _{s \in S} v\left(\ln u_{s}, n\right) .
$$

Proof. For every integer $k \geq 0$, set $\varepsilon_{k}=\sum_{n \geq k} \sup _{s \in S} v\left(\ln u_{s}, n\right)$. Consider the cone $\mathcal{C}$ of strictly positive continuous functions $f$ such that $v(\ln f, k)<\varepsilon_{k}$. For $x_{0} \in E$, the section $\mathcal{C} \cap\left\{f: f\left(x_{0}\right)=1\right\}$ is an equicontinuous and bounded set of continuous functions. Therefore, the cone $\mathcal{C}$ has a compact base.

This cone is left invariant by $P$, since we have, for every $x, y \in E$ satisfying $d(x, y) \leq \eta_{k}$ :

$$
\begin{aligned}
& P f(y)=\sum_{s \in S} u_{s}(y) f(s y)=\sum_{s \in S} \frac{u_{s}(y)}{u_{s}(x)} \frac{f(s y)}{f(s x)} u_{s}(x) f(s x) \\
& \leq \sum_{s \in S} \mathrm{e}^{\sup _{s \in S} v\left(\ln u_{s}, k\right)} \mathrm{e}^{\varepsilon_{k+1}} u_{s}(x) f(s x)=\mathrm{e}^{\varepsilon_{k}} \operatorname{Pf}(x) .
\end{aligned}
$$

This gives the inequality;

$$
v(\ln P f, k) \leq v(\ln f, k+1)+w(k)
$$

From Schauder-Tychonov theorem, we deduce the existence of a proper function $h$ for $P$ in the cone $\mathcal{C}$.

Assuming (16) and the functions $u_{s}$ strictly positive, let $h$ given by the previous lemma such that $P h=\lambda h$. The conditions (11) and (12) are satisfied by $\lambda^{-1} P$ (see inequality (8)). The relativised operator is defined by

$$
{ }^{h} P f(x)=\frac{1}{\lambda h(x)} P(h f)(x) .
$$


We have strong proximality and, for $f \in C(E)$, the sequence $\left(\left({ }^{h} P\right)^{n} f\right)_{n \geq 0}$ converges uniformly to $\nu(f)$ where $\nu$ is the unique ${ }^{h} P$-invariant probability on $E$. The sequence $\left(\lambda^{-n} P^{n} f\right)_{n \geq 0}$ converges uniformly to $\nu\left(h^{-1} f\right) h$. The measure $\tilde{\nu}=\left(\nu\left(\frac{1}{h}\right) h\right)^{-1} \nu$ is the unique $\lambda^{-1} P$-invariant probability.

Some partial results can also be proved when the weights are not supposed to be strictly positive:

Theorem 3.5. If $P$ (power bounded) satisfies

$$
\sum_{n \geq 0} w(n, 0)<+\infty
$$

and $\rho(P)=\lim _{n}\left(\left\|P^{n} 1\right\|_{\infty}\right)^{1 / n}=1$, there exists a continuous non negative function $h$ which is $P$-invariant and not identically 0 . If $\left(S,\left(u_{s}\right)_{s \in S}\right)$ is proximal, then $h$ is strictly positive.

Proof. Since $P$ satisfies the hypotheses of Theorem 1.1, we know that the sequence $\left(\frac{1}{n} \sum_{k=0}^{n-1} P^{k} 1\right)_{n \geq 1}$ converges uniformly on $E$ to a non-negative continuous $P$-invariant function $h$.

If $h$ is the null function, then $W_{1}$ is reduced to zero and $\lim _{n \rightarrow+\infty}\left\|P^{n} 1\right\|_{\infty}=0$. This is impossible if there exists a $P$-invariant probability measure $\nu$. Let us show the existence of such a measure under our assumptions.

We consider the following operators:

$$
P_{\varepsilon} f(x)=\sum_{s \in S}\left(u_{s}(x)+\varepsilon_{s}\right) f(s x),
$$

where $\left(\varepsilon_{s}\right)_{s \in S}$ is a family of strictly positive reals such that $\sum_{s} \varepsilon_{s}<\infty$. If (18) holds, then $P_{\varepsilon}$ satisfies the conditions of Lemma 3.4 (we have: $v\left(\ln \left(u_{s}+\varepsilon_{s}\right), k\right) \leq \varepsilon_{s}^{-1} v\left(u_{s}, k\right)$ ).

Therefore, there exist a strictly positive continuous function $h_{\varepsilon}$ and a scalar $\lambda_{\varepsilon}$ such that $P_{\varepsilon} h_{\varepsilon}=\lambda_{\varepsilon} h_{\varepsilon}$. We have $1 \leq C_{\varepsilon} h_{\varepsilon}$, for some finite constant $C_{\varepsilon}$. Moreover there exists a measure $\nu_{\varepsilon}$ such that $P_{\varepsilon} \nu_{\varepsilon}=\lambda_{\varepsilon} \nu_{\varepsilon}$ (since $h_{\varepsilon}>0$, the eigenvalue is the same for the action of $P$ on functions and on measures). The inequalities $P^{n} 1 \leq P_{\varepsilon}^{n} 1 \leq C P_{\varepsilon}^{n} h_{\varepsilon}=C_{\varepsilon} \lambda_{\varepsilon}^{n} h_{\varepsilon}$ and the hypothesis $\rho(P)=1$ imply: $\lambda_{\varepsilon} \geq 1$.

Letting the constants $\varepsilon_{s}$ tend to 0 for each $s \in S$ and taking a cluster value of the family $\left\{\nu_{\varepsilon}, \varepsilon>0\right\}$, we get a probability measure $\nu_{0}$ such that $P \nu_{0}=\lambda_{0} \nu_{0}$, for a constant $\lambda_{0} \geq 1$, and therefore $\lambda_{0}=1$ since $P$ is power bounded.

\section{Convergence of $\sum P^{n} f$ (Weaker hypotheses on $f$ )}

In this section, the assumptions are stronger for the weights $u_{s}$, but weaker for the spaces of functions $f$ that we consider.

\subsection{Hypotheses and notations}

We assume that the functions $u_{s}, s \in S$, are strictly positive and satisfy

$$
\sum_{k \geq 0} \sup _{s \in S} v\left(\ln u_{s}, k\right)<+\infty .
$$

By Lemma 3.4 there exist $\lambda>0$, a strictly positive continuous function $h$ satisfying $P h=\lambda h$ and an unique $\lambda^{-1} P$-invariant probability $\nu$ on $E$ such that, for all $f \in C(E)$, the sequence of functions $\left(\lambda^{-n} P^{n} f\right)_{n \geq 0}$ converges uniformly to $\nu(f) h$. Without loss of generality, in the following we will suppose that the support of the measure $\nu$ is equal to $E$. Replacing $P$ with $\lambda^{-1} P$, we can also assume $\lambda=1$, so that $P$ is power bounded, $\nu$ is $P$-invariant and $\|P f\|_{1} \leq\|f\|_{1}$, for $f \in L^{1}(\nu)$.

We introduce now a condition for the regularity of functions on $E$ which is weaker than that defined in (2.2). 
Notations. For $n$ integer $\geq 0, x \in E, f$ Borel function $f$ on $E$, we define:

$$
\begin{aligned}
\tilde{\mathcal{V}}(f, x, n) & =\|f(x)-f(\cdot)\|_{L^{\infty}\left(B\left(x, \eta_{n}\right), \nu\right)}, \\
\widetilde{v}(f, n) & =\int_{E} \tilde{\mathcal{V}}(f ; x, n) \nu(\mathrm{d} x) .
\end{aligned}
$$

We set

$$
\begin{aligned}
V & =\left\{f \in L^{\infty}(E, \nu): \lim _{n \rightarrow+\infty} \tilde{v}(f, n)=0\right\}, \\
W_{2} & =\left\{f \in V: \lim _{n \rightarrow+\infty}\left\|P^{n} f\right\|_{\infty}=0\right\} .
\end{aligned}
$$

Choose a function $\varphi$ from $\mathbb{N}$ to $] 0,+\infty[$ such that

$$
\sum_{k \geq 0} \varphi(k)=+\infty \text { and } \sum_{k \geq 0} \varphi(k)\left(\sum_{n \geq k} \sup _{s \in S} v\left(\ln u_{s}, n\right)\right)<+\infty .
$$

We may then define the analogous of the quantities introduced in (2.2):

$$
\begin{aligned}
|f|_{\varphi, n}^{\prime} & =\sum_{k \geq 0} \varphi(k) \widetilde{v}(f, k+n), n \geq 0 \\
|f|_{\varphi}^{\prime} & =|f|_{\varphi, 0}^{\prime}=\sum_{k \geq 0} \varphi(k) \widetilde{v}(f, k) .
\end{aligned}
$$

Let $\tilde{B}_{\varphi}$ be the subspace of $L^{1}(E, \nu)$ defined by $\tilde{B}_{\varphi}=\left\{f \in L^{1}(E, \nu):|f|_{\varphi}^{\prime}<+\infty\right\}$. We will see that $\left(\tilde{B}_{\varphi},\|\|_{1},\left(||_{\varphi, n}^{\prime}\right)_{n \geq 0}\right)$ satisfies the hypotheses of Theorem 1.1.

Theorem 4.1. Under the hypotheses (19), we have $V=\mathbb{C} h \oplus W_{2}$.

Let $r \in \mathbb{N}$. Suppose that $\sum_{n \geq 0} n^{r} \sup _{s \in S} v\left(\ln u_{s}, n\right)<+\infty$. Then, for all $f \in W_{2}$ such that $\sum_{n \geq 0} n^{r} \tilde{v}(f, n)<$ $+\infty$, we have $\sum_{n \geq 0} n^{r}\left\|P^{n} f\right\|_{\infty}<+\infty$.

The proof follows from several lemmas.

Lemma 4.2. There exists a positive real number c such that, for all $f \in \mathcal{L}^{\infty}(E, \nu)$,

$$
\|f\|_{L^{\infty}} \leq c\left(\tilde{v}(f, 0)+\|f\|_{1}\right) \leq c\left((\varphi(0))^{-1}|f|_{\varphi}^{\prime}+\|f\|_{1}\right) .
$$

Proof. For $x \in E$ and $r>0$, we denote by $B(x, r)$ the open ball $\{y \in E: d(x, y)<r\}$. Let $f \in \mathcal{L}^{1}(E, \nu)$. For all $y \in E$ and for $\nu$-almost all $x \in E$, we have:

$$
\begin{aligned}
1_{B\left(x, \eta_{0}\right)}(y)|f(x)| & \leq 1_{B\left(x, \eta_{0}\right)}(y)(|f(x)|-|f(y)|)+|f(y)| \\
& \leq\|f(\cdot)-f(y)\|_{L^{\infty}\left(E, B\left(y, \eta_{0}\right), \nu\right)}+|f(y)| .
\end{aligned}
$$

After integration we obtain, for $\nu$-almost all $x \in E$,

$$
\nu\left(B\left(x, \eta_{0}\right)\right)|f(x)| \leq \tilde{v}\left(f, \eta_{0}\right)+\|f\|_{1} \leq(\varphi(0))^{-1}|f|_{\varphi}+\|f\|_{1} .
$$


To conclude we have to show that $\inf _{x \in E} \nu\left(B\left(x, \eta_{0}\right)\right)>0$. Let $\left(x_{n}\right)_{n \geq 0}$ be a sequence of elements of $E$ such that $\lim _{n \rightarrow+\infty} \nu\left(B\left(x_{n}, \eta_{0}\right)\right)=\inf _{x \in E} \nu\left(B\left(x, \eta_{0}\right)\right)$. From the compactness of $E$, we can suppose that the sequence $\left(x_{n}\right)_{n \geq 0}$ converges to an element $x$ of $E$. But then, as the support of $\nu$ is equal to $E$, we have

$$
\liminf _{n \rightarrow+\infty} \nu\left(B\left(x_{n}, \eta_{0}\right)\right) \geq \nu\left(B\left(x, \eta_{0}\right)\right)>0 .
$$

Lemma 4.3. The set $\mathcal{C}_{1}=\left\{f \in L^{1}(E, \nu):|f|_{\varphi}^{\prime}+\|f\|_{1} \leq 1\right\}$ is a compact subset of $L^{1}(E, \nu)$.

Proof. For $k \geq 0$ consider a finite open covering $\left\{B\left(x_{j, k}, \eta_{k}\right), 1 \leq j \leq q_{k}\right\}$ of $E$ extracted from the open covering $\left\{B\left(x, \eta_{k}\right), x \in E\right\}$. Let $\left(\psi_{j, k}\right)_{1 \leq j \leq p_{k}}$ be a partition of unity subordinate to this covering (i.e. the functions $\psi_{j, k}, k \geq 0,1 \leq j \leq p_{k}$, are non-negative continuous and satisfy

$$
\forall x \in E, \sum_{j=1}^{k} \psi_{j, k}(x)=1 \text { and } \forall x \notin B\left(x_{j, k}, \eta_{k}\right), \psi_{j, k}(x)=0 .
$$

Let $\left(f_{n}\right)_{n \geq 0}$ be a sequence of functions of $\mathcal{C}_{1}$. By Lemma 4.2, these functions are bounded by $c\left((\varphi(0))^{-1}+1\right)$. Since the sequence $\left(\widetilde{v}\left(f_{p}, n\right)\right)_{n \geq 0}$ is decreasing, we have:

$$
\left(\sum_{k=0}^{n} \varphi(k)\right) \widetilde{v}\left(f_{p}, n\right) \leq \sum_{k=0}^{n} \varphi(k) \widetilde{v}\left(f_{p}, k\right) \leq\left|f_{p}\right|_{\varphi}^{\prime} \leq 1
$$

and we get, for all integers $k, n$ and $p$,

$$
\begin{aligned}
\left\|f_{n+p}-f_{n}\right\|_{1}= & \sum_{j=1}^{p_{k}} \int_{E} \psi_{j, k}\left|f_{n+p}-f_{n}\right| \mathrm{d} \nu \\
\leq & \sum_{j=1}^{p_{k}}\left(\int_{E} \psi_{j, k}\left|f_{n+p}-f_{n+p}\left(x_{j, k}\right)\right| \mathrm{d} \nu\right. \\
& \left.+\int_{E} \psi_{j, k}\left|f_{n}-f_{n}\left(x_{j, k}\right)\right| \mathrm{d} \nu+\int_{E} \psi_{j, k} \mathrm{~d} \nu\left|f_{n+p}\left(x_{j, k}\right)-f_{n}\left(x_{j, k}\right)\right|\right) \\
\leq & \sum_{j=1}^{p_{k}}\left(\int_{E} \psi_{j, k} \widetilde{\mathcal{V}}\left(f_{n+p}, \cdot, k\right) \mathrm{d} \nu+\int_{E} \psi_{j, k} \widetilde{\mathcal{V}}\left(f_{n}, \cdot, k\right) \mathrm{d} \nu+\int_{E} \psi_{j, k} \mathrm{~d} \nu\left|f_{n+p}\left(x_{j, k}\right)-f_{n}\left(x_{j, k}\right)\right|\right) \\
\leq & \widetilde{v}\left(f_{n+p}, k\right)+\widetilde{v}\left(f_{n}, k\right)+\sum_{j=1}^{p_{k}} \int_{E} \psi_{j, k} \mathrm{~d} \nu\left|f_{n+p}\left(x_{j, k}\right)-f_{n}\left(x_{j, k}\right)\right| \\
\leq & 2\left(\sum_{j=0}^{k} \varphi(j)\right)^{-1}+\sum_{j=1}^{p_{k}} \int_{E} \psi_{j, k} \mathrm{~d} \nu\left|f_{n+p}\left(x_{j, k}\right)-f_{n}\left(x_{j, k}\right)\right| .
\end{aligned}
$$

If $(\sigma(n))_{n \geq 0}$ is a strictly increasing sequence of integers such that the real sequences $\left(f_{\sigma(n)}\left(x_{j, k}\right)\right)_{n \geq 0}$ converge, for every $k \geq 0$ and $j \in\left[1, p_{k}\right]$, then the sequence $\left(f_{\sigma(n)}\right)_{n \geq 0}$ is a Cauchy sequence in $L^{1}(E, \nu)$.

This shows that the set $\mathcal{C}_{1}$ is relatively compact in $L^{1}(E, \nu)$. It remains to show that $\mathcal{C}_{1}$ is closed. 
Let $\left(f_{n}\right)_{n \geq 0}$ be a sequence of functions of $\mathcal{C}_{1}$ which converges in $L^{1}(E, \nu)$ to a function $f$. Taking if necessary a subsequence, we can assume that the sequence $\left(f_{n}\right)_{n>0}$ converges $\nu$-a.e. to $f$. From Egorov's theorem, there exists an increasing sequence $\left(E_{p}\right)_{p \geq 0}$ of Borel sets in $E$ such that

$$
\lim _{p \rightarrow+\infty} \nu\left(E_{p}\right)=1 \quad \text { and } \quad \forall p \geq 0, \lim _{n \rightarrow+\infty}\left\|f_{n}-f\right\|_{L^{\infty}\left(E_{p}, \nu\right)}=0 .
$$

For every integer $p \geq 0$ and every integer $N \geq 1$, we have then:

$$
\begin{aligned}
& \left.\sum_{k=0}^{N} \varphi(k) \int_{E_{p}}\|f(x)-f(\cdot)\|_{L^{\infty}\left(B\left(x, \eta_{k}\right) \cap E_{p}, \nu\right.}\right) \\
= & \lim _{n \rightarrow+\infty} \sum_{k=0}^{N} \varphi(k) \int_{E_{p}}\left\|f_{n}(x)-f_{n}(\cdot)\right\|_{L^{\infty}\left(B\left(x, \eta_{k}\right) \cap E_{p}, \nu\right)} \nu(\mathrm{d} x) \\
\leq & \sup _{n \geq 0}\left|f_{n}\right|_{\varphi}^{\prime} \leq 1 .
\end{aligned}
$$

Letting $p$, then $N$, tend to $+\infty$, we get: $|f|_{\varphi}^{\prime} \leq 1$. This implies the result.

Lemma 4.4. If $f$ is a function on $E$ and $k$ an integer $\geq 0$, we have:

$$
\widetilde{v}(P f, k) \leq \mathrm{e}^{z(k)} \widetilde{v}(f, k+1)+z(k)\|f\|_{1},
$$

where

$$
z(k)=\sup _{s \in S} \mathrm{e}^{v\left(\ln u_{s}, k\right)} v\left(\ln u_{s}, k\right)
$$

Proof. Let $n$ be $\in \mathbb{N}$ and $(x, y) \in E^{2}$ such that $d(x, y) \leq \eta_{k}$. We have:

It follows that

$$
\left|1-\frac{u_{s}(y)}{u_{s}(x)}\right| \leq \mathrm{e}^{\left|\ln \frac{u_{s}(x)}{u_{s}(y)}\right|}\left|\ln \frac{u_{s}(x)}{u_{s}(y)}\right| \leq \mathrm{e}^{v\left(\ln u_{s}, k\right)} v\left(\ln u_{s}, k\right) \leq z(k) .
$$

$$
\begin{aligned}
|P f(x)-P f(y)| & \leq \sum_{s \in S}\left|u_{s}(x) f(s x)-u_{s}(y) f(s y)\right| \\
& \leq \sum_{s \in S} u_{s}(y)|f(s x)-f(s y)|+\sum_{s \in S}\left|u_{s}(x)-u_{s}(y)\right||f(s x)| \\
& \leq(1+z(k)) \sum_{s \in S} u_{s}(x)|f(s x)-f(s y)|+z(k) P|f|(x) \\
& \leq \mathrm{e}^{z(k)} \sum_{s \in S} u_{s}(x) \mathcal{V}(f, s x, k+1)+z(k) P|f|(x) .
\end{aligned}
$$

We obtain the result by integrating with respect to $\nu$ and using the invariance of $\nu$ by $P$. 
Corollary 4.5. There exists a positive real $C$ such that, for every $f \in \tilde{B}_{\varphi}$ :

$$
\forall n \geq 1, \forall k \geq 0,\left|P^{n} f\right|_{\varphi, k}^{\prime} \leq C\left(|f|_{\varphi, n+k}^{\prime}+\sum_{j=0}^{n-1} a_{n+k-j-1}\left\|P^{j} f\right\|_{1}\right)
$$

where $a_{n}=\sum_{j \geq 0} \varphi(j) \sup _{s \in S} v\left(\ln u_{s}, j+n\right)$

Proof. From Lemma 4.4 we obtain

$$
\forall k \geq 0,|P f|_{\varphi, k}^{\prime} \leq \mathrm{e}^{z(k)}|f|_{\varphi, k+1}^{\prime}+b_{k}\|f\|_{1}
$$

with $b_{k}=\sum_{j \geq 0} \varphi(j) z(k+j) \leq \mathrm{e}^{\sup _{s \in S} v\left(\ln u_{s}, 0\right)} a_{k}$. From this inequality applied to the functions $P^{n-1} f$, $P^{n-2} f, \ldots, f$, we get, for all $k \geq 0$ and $n \geq 1$,

$$
\left|P^{n} f\right|_{\varphi, k}^{\prime} \leq \mathrm{e}^{z(k)+\ldots+z(k+n-1)}|f|_{\varphi, k+n}^{\prime}+\sum_{j=0}^{n-1} \mathrm{e}^{z(k)+\ldots+z(k+n-j-2)} b_{k+n-j-1}\left\|P^{j} f\right\|_{1}
$$

Setting $C=\mathrm{e}^{\sum_{j \geq 0} z(j)} \max \left\{1, \mathrm{e}^{\sup _{s \in S} v\left(\ln u_{s}, 0\right)}\right\}$, we get $(23)$.

\subsection{Proof of Theorem $\mathbf{4 . 2}$}

Proof. We know that, for $f \in C(E)$, the sequence of functions $\left(P^{n} f\right)_{n \geq 0}$ converges uniformly to $\nu(f) h$ and therefore, for $f \in L^{1}(E, \nu)$, the sequence $\left(P^{n} f\right)_{n \geq 0}$ converges in $L^{1}(E, \nu)$ to $\nu(f) h$.

Let $f \in V$. As $\lim _{n \rightarrow+\infty} \tilde{v}(f, n)=0$, we can choose a function $\varphi$ from $\mathbb{N}$ to $] 0,+\infty\left[\right.$ such that $f \in \tilde{B}_{\varphi}$ and the conditions (22) are satisfied.

We can substract $\nu(f) h$ from $f$ to have $\nu(f)=0$ (if $\nu(f) \neq 0, \lim _{n} \frac{1}{n} \sum_{k=0}^{n-1} P^{k} f$ is proportional to $h$ and $h$ is in the space $V)$.

From Lemmas 4.3 and $23,\left(\tilde{B}_{\varphi}, P,\|\|_{1},\left(||_{n}\right)_{n \geq 0}\right)$ verifies the hypotheses of Theorem 1.1. It follows that $f$ is in $W_{2}^{\varphi}$, where $W_{2}^{\varphi}=\left\{f \in V: \lim _{n \rightarrow+\infty}\left(\left|P^{n} f\right|_{\varphi}^{\prime}+\left\|P^{n} f\right\|_{1}\right)=0\right\}$. From Lemma 4.2, we deduce that $f$ belongs to $W_{2}=\left\{g \in V: \lim _{n \rightarrow+\infty}\left\|P^{n} g\right\|_{\infty}=0\right\}$. Therefore we have: $V=\mathbb{C} h \oplus W_{2}$.

Let $f$ such that $\sum_{n \geq 0} n^{r} \tilde{v}(f, n)<+\infty$. There exists a function $\varphi$ from $\mathbb{N}$ into $] 0,+\infty[$ such that $(22)$ and

$$
\sum_{n \geq 0} \varphi(n) \sum_{k \geq n} k^{r} \tilde{v}(f, k)<\infty
$$

From Theorem 1.1, we have then

$$
\sum_{n \geq 0} n^{r}\left(\left|P^{n} f\right|_{\varphi}^{\prime}+\left\|P^{n} f\right\|_{1}\right)<\infty
$$

which implies by Lemma 4.2 :

$$
\sum_{n \geq 0} n^{r}\left\|P^{n} f\right\|_{\infty}<\infty .
$$




\section{Application to the Clt for some Markov chains}

5.1 .

We consider a Markovian operator $P$ of the form (5). Theorem 3.2 gives sufficient conditions for a function $f$ to be of the form

$$
f=g-P g .
$$

This will be applied to obtain the central limit theorem.

Let $\left(\Omega=E^{\mathbb{N}}, \mathcal{B}(\Omega),\left(X_{n}\right)_{n \geq 0},\left(\mathbb{P}_{x}\right)_{x \in E}\right)$ be the canonical Markov chain with transition probability $P$. Let $x$ be an element of $E$ and $f$ a function defined on $E$. One says that the process $\left(f\left(X_{n}\right)\right)_{n \geq 0}$ satisfies the central limit theorem (CLT) under $\mathbb{P}_{x}$ if, for a constant $\sigma>0$, the sequence of real random variables $\left(\frac{1}{\sqrt{n}}\left(f\left(X_{0}\right)+\cdots+\right.\right.$ $\left.\left.f\left(X_{n-1}\right)\right)\right)_{n \geq 1}$, defined on $\left(\Omega, \mathcal{B}(\Omega), \mathbb{P}_{x}\right)$, converges in law to the normal law $\mathcal{N}\left(0, \sigma^{2}\right)$.

Using the representation (24) we can apply the method introduced by Gordin [7], Gordin and Lifvsic [8], to reduce the process $\left(f\left(X_{n}\right)\right)$ to a martingale difference $(c f .[3,10])$ to prove the CLT. Strengthening the conditions on $P$ and $f$, we can apply a result of Rio [22] for weakly dependant sequences ( $c f$. also [13]) and get the optimal rate of convergence in the CLT.

In the following theorem we use the notations of Section 3 and Theorem 3.3iv).

Theorem 5.1. Let us assume hypotheses (3.1) and the family $\left\{u_{s}: s \in S\right\}$ strongly proximal. Let $\nu$ be the unique $P$-invariant probability on $E$ and $f$ be a continuous function on $E$ such that $\nu(f)=0$ and $\nu(|f|)>0$.

i) If $\sum_{k>0} v(f, k)<\infty$, then for all $x \in E$ the sequence $\left(f\left(X_{n}\right)\right)_{n \geq 0}$ satisfies, under the probability $\mathbb{P}_{x}$, the central limit theorem and the functional central limit theorem.

ii) If $\sum_{k \geq 0} k w(k, 0)<+\infty$ and $\sum_{k \geq 0} k v(f, k)<+\infty$, then for all $x \in E$ the sequence $\left(f\left(X_{n}\right)\right)_{n \geq 0}$ satisfies, under the probability $\mathbb{P}_{x}$, the central limit theorem at a rate in $O\left(\frac{1}{\sqrt{n}}\right)$.

Proof. $i)$ Theorem 3.2 and condition $\sum_{k \geq 0} v(f, k)<\infty$ imply $\sum_{n \geq 0}\left\|P^{n} f\right\|_{\infty}<+\infty$.

Setting $g=\sum_{n \geq 0} P^{n} f$, we obtain a continuous function such that $f=g-P g$. The sums $f\left(X_{0}\right)+\cdots+f\left(X_{n-1}\right)$ can be written: $S_{n}+g\left(X_{0}\right)-g\left(X_{n}\right)$ with $S_{n}=\sum_{k=0}^{n-1}\left[g\left(X_{k+1}\right)-P g\left(X_{k}\right)\right]$.

As $g\left(X_{0}\right)-g\left(X_{n}\right)$ remains bounded, we can replace the sums $f\left(X_{0}\right)+\cdots+f\left(X_{n-1}\right)$ with $S_{n}$. Denote $Y_{n}=g\left(X_{n}\right)-P g\left(X_{n-1}\right), n \geq 1$. Let $x$ be an element of $E$.

For the probability $\mathbb{P}_{x}$ the sequence $\left(Y_{n}\right)_{n \geq 1}$ is a sequence of martingale increments with respect to the natural filtration $\left(\mathcal{F}_{n}\right)_{n \geq 0}$ associated to the process $\left(X_{n}\right)_{n \geq 0}$. According to Brown [3], the first assertion of Theorem 5.1 follows from the two conditions:

$$
\begin{aligned}
& \text { 1) } \forall \varepsilon>0, \lim _{n \rightarrow+\infty} \frac{1}{\sigma_{n}^{2}} \sum_{k=1}^{n} \mathbb{E}_{x}\left[Y_{k}^{2} 1_{\left\{\left|Y_{k}\right|>\varepsilon \sigma_{n}\right\}}\right]=0, \\
& \text { 2) } \sigma_{n}^{-2} V_{n} \stackrel{\mathbb{P}_{x}}{\longrightarrow} 1
\end{aligned}
$$

where

$$
\sigma_{n}^{2}=\sum_{k=1}^{n} \mathbb{E}_{x}\left[Y_{k}^{2}\right] \text { and } V_{n}=\sum_{k=1}^{n} \mathbb{E}_{x}\left[Y_{k}^{2} \mid \mathcal{F}_{k-1}\right] .
$$

The first condition is satisfied because $g$ is a bounded function. On the other hand we have:

$$
\sigma_{n}^{2}=\sum_{k=1}^{n} \mathbb{E}_{x}\left[g^{2}\left(X_{k}\right)-(P g)^{2}\left(X_{k-1}\right)\right]=\sum_{k=1}^{n} P^{k-1}\left(P g^{2}(x)-(P g)^{2}\right)(x) .
$$


We know that, for each continuous function $h$, the sequence of continuous functions $\left(P^{n} h\right)_{n \geq 0}$ converges uniformly on $E$ to $\nu(h)$. It follows that the sequence $\left(\frac{1}{n} \sigma_{n}^{2}\right)_{n \geq 1}$ converges to $\sigma^{2}=\int_{E}\left(P\left(g^{2}\right)-(P g)^{2}\right) \mathrm{d} \nu$.

If $\sigma=0$ then, for $\nu$-almost all $x \in E$, we have: $P^{k}(g-P g(x))^{2}(x)=0, \forall k \geq 0$, and therefore $g$ is constant on the trajectories $s_{n} \cdots s_{1} x$ of $x$. The proximality implies that $f=0, \nu$-a.e.

We have $V_{n}=\sum_{k=1}^{n}\left(P g^{2}-(P g)^{2}\right)\left(X_{k-1}\right)$. By the law of large numbers for martingale increments, we know that, for each continuous function $h$ on $E$, the sequence $\left(\frac{1}{n} \sum_{k=1}^{n}\left(h\left(X_{k}\right)-P h\left(X_{k-1}\right)\right)\right)$ converges $\mathbb{P}_{x^{-}}$a.e. to zero. As $C(E)$ is separable and $P$ is a contraction of $\left(C(E),\|\|_{\infty}\right)$, for $\mathbb{P}_{x}$-a.e. $\omega \in \Omega$, the sequence $\left(\frac{1}{n} \sum_{k=1}^{n}\left(h\left(X_{k}(\omega)\right)-P h\left(X_{k-1}(\omega)\right)\right)\right.$ converges to zero, for all $h \in C(E)$. If $\lambda_{\omega}$ is a limit point for the weak convergence of the sequence of probabilities $\left(\frac{1}{n} \sum_{k=1}^{n} P^{k-1}(x, \cdot)\right)_{n \geq 1}$, we have, for $\mathbb{P}_{x}$-almost all $\omega \in \Omega, \lambda_{\omega}(h-$ $P h)=0$, for all $h \in C(E)$. The measure $\lambda_{\omega}$ is $P$-invariant and therefore $\lambda_{\omega}=\nu$.

This proves that, for $\mathbb{P}_{x}$-almost all $\omega \in \Omega$, for all $h \in C(E)$, the sequence $\left(\frac{1}{n} \sum_{k=1}^{n} h\left(X_{k}(\omega)\right)\right)_{n \geq 1}$ converges to $\nu(h)$; therefore the sequence $\left(\frac{V_{n}}{n}\right)_{n \geq 1}$ converges $\mathbb{P}_{x}$-a.e. to $\sigma^{2}$ and the condition 2$)$ is satisfied.

ii) To prove the second assertion of the theorem, we apply a theorem of Rio [22] (see [13] for a formulation of this theorem adapted to our frame). It is sufficient to prove that, for all non negative integers $p_{1}, p_{2}$ and $p_{3}$ such that $p_{1}+p_{2}+p_{3} \leq 3, \sum_{n \geq 0} n \psi(n)<+\infty$, where $\psi=\psi_{p_{1}, p_{2}, p_{3}}$ is the sequence defined by

$$
\left.\psi(n)=\sup _{\alpha_{2}, \alpha_{3} \in \mathbb{N}} \| P^{n}\left(f^{p_{1}}\left(P^{\alpha_{2}} f^{p_{2}}\left(P^{\alpha_{3}} f^{p_{3}}\right)\right)\right)-\nu\left(f^{p_{1}} P^{\alpha_{2}} f^{p_{2}}\left(P^{\alpha_{3}} f^{p_{3}}\right)\right)\right) \|_{\infty}
$$

We have

$$
\begin{aligned}
\psi(n) \leq & \sup _{\alpha_{2}, \alpha_{3} \in \mathbb{N}}\left(\left\|Q^{n}\left(f^{p_{1}}\left(Q^{\alpha_{2}} f^{p_{2}}\left(Q^{\alpha_{3}} f^{p_{3}}\right)\right)\right)\right\|_{\infty}\right. \\
& \left.+\|f\|_{\infty}^{p_{3}}\left\|Q^{n}\left(f^{p_{1}}\left(Q^{\alpha_{2}} f^{p_{2}}\right)\right)\right\|_{\infty}\right)+\|f\|_{\infty}^{p_{2}+p_{3}}\left\|Q^{n} f^{p_{1}}\right\|_{\infty} .
\end{aligned}
$$

Let $f \in C(E)$ such that $\sum_{k \geq 0} k v(f, k)<+\infty$. Choose a function $\varphi$ from $\mathbb{N}$ to $] 0,+\infty[$ such that

$$
\begin{gathered}
\sum_{n \geq 0} \varphi(n)=+\infty, \sum_{n \geq 0} \varphi(n)\left(\sum_{k \geq n} k w(k, 0)\right)<+\infty \\
\text { and } \sum_{n \geq 0} \varphi(n)\left(\sum_{k \geq n} k v(f, k)\right)<+\infty .
\end{gathered}
$$

The space $B_{\varphi}$ equipped with the norm \|\|$_{\infty}$ and the sequence of semi-norms $(||)_{n \geq 0}$ satisfies the hypotheses of Lemma 1.2 with $\delta(n)=\sum_{k \geq 0} \varphi(k) w(n+k, 0)$. The condition $\sum_{n \geq 0} n \psi(n)<+\infty$ is then a consequence of the following conditions (with the notations of Lems. 1.2 and 13):

$$
\sum_{n \geq 0} n|f|_{\varphi, n}<+\infty, \sum_{n \geq 0} n \delta(n)<+\infty \text { and } \sum_{n \geq 0} n a(0, n)<\infty
$$

These conditions are equivalent respectively to:

$$
\begin{gathered}
\sum_{k \geq 0} \varphi(k) \sum_{n \geq k} n v(f, n)<+\infty, \quad \sum_{k \geq 0} \varphi(k) \sum_{n \geq k} n w(n, 0)<+\infty, \\
\sum_{k \geq 0} \varphi(k) \sum_{n \geq k} n w(0, n)<+\infty .
\end{gathered}
$$

The result follows. 
We assume now the hypotheses (19). Let us remark that $\left(\tilde{B}_{\varphi}, P,\|\|_{1},\left(||_{n}\right)_{n \geq 0}\right)$ satisfies the hypotheses of Lemma 1.2 except that $\left(\tilde{B}_{\varphi},\|\|_{1}\right)$ is not an normed algebra. But $\tilde{B}_{\varphi}$ is contained in $L^{\infty}(E, \nu)$ and we have $\|f g\|_{1} \leq\|f g\|_{\infty} \leq\|f\|_{\infty}\|g\|_{\infty}$. Then it is easy to see that we can apply Lemma 1.2. Therefore, we have:

Theorem 5.2. Let us assume (19). Let $f$ be a (non null) function in $L^{\infty}(E, \nu)$ such that $\nu(f)=0$.

i) If we have $\sum_{k \geq 0} \tilde{v}(f, k)<\infty$, then for $\nu$-almost all $x \in E$ the sequence $\left(f\left(X_{n}\right)\right)_{n \geq 0}$ satisfies, under the probability $\mathbb{P}_{x}$, the central limit theorem and the functional central limit theorem.

ii) If $\sum_{k \geq 0} k \sup _{s \in S} v\left(\ln u_{s}, k\right)<+\infty$ and $\sum_{k \geq 0} k \tilde{v}(f, k)<+\infty$, then for $\nu$-almost all $x \in E$ the sequence $\left(f\left(X_{n}\right)\right)_{n \geq 0}$ satisfies, under the probability $\mathbb{P}_{x}$, the central limit theorem at the rate $O\left(\frac{1}{\sqrt{n}}\right)$.

\section{Application to dynamical Systems: CLT, Borel-CANtelli Property}

\subsection{Hypotheses and notations}

In this section, we suppose that $P$ is Markovian. We assume that there exists a surjective application $\tau$ from $E$ onto $E$ leaving invariant a $P$-invariant probability measure $\nu$ and such that the operator $P$ is the dual of the operator $T$ of composition by $\tau$ :

$$
\int_{E} P f g \mathrm{~d} \nu=\int_{E} f g \circ \tau \mathrm{d} \nu, \forall f, g \in L^{2}(E, \nu)
$$

We consider the dynamical system $(E, \mathcal{E}, \nu, \tau)$.

\section{- Central limit theorem}

As in the case of Markov chains, following the method introduced by Gordin [7], using the representation (24) of $f$ we can apply martingale methods to the stationary sequence $\left(T^{n} f\right)$ (with respect to the measure $\nu$ ).

Recall that two functions $f$ and $g$ are "homologous" for the dynamical system $(E, \mathcal{E}, \nu, \tau)$, if there exists a mesurable $\psi$ such that $f=g+T \psi-\psi$. A function $f$ is a coboundary if it is homologous to 0 .

Theorem 6.1. Let us assume (3.1) and the family $\left\{u_{s}: s \in S\right\}$ strongly proximal. Let $f$ be a continuous function on $E$ such that $\nu(f)=0$ and $f$ is not a coboundary.

i) If the condition $\sum_{k \geq 0} v(f, k)<\infty$ holds, the function $f$ is homologous in the dynamical system $(E, \mathcal{E}, \nu, \tau)$ to a function generating a sequence of reverse martingale increments. The sequence $\left(T^{n} f\right)_{n \geq 0}$ satisfies the central limit theorem and the functional limit theorem.

ii) If $\sum_{k \geq 0} k w(k, 0)<+\infty$ and $\sum_{k \geq 0} k v(f, k)<+\infty$, then the sequence $\left(T^{n} f\right)$ satisfies the central limit theorem at the rate $O\left(\frac{1}{\sqrt{n}}\right)$.

We have the same conclusions if we replace the hypotheses (3.1) with the hypotheses (19) and $v(k, f)$ by $\tilde{v}(f, k)$.

Proof. From Theorem 3.2, there exists $g \in C(E)$ such that $f=g-P g$.

We can write then $f=g_{1}+h-T h$, with $g_{1}=g-T P g$ and $h=-P g$. The function $f$ is homologous to $g_{1}$. The relation $P g_{1}=0$ shows that the sequence $\left(T^{n} g_{1}\right)$ is a sequence of reverse martingale increments with respect to the decreasing filtration $\left(\tau^{-n} \mathcal{B}(E)\right)_{n \geq 0}$. This stationary ergodic sequence satisfies therefore the central limit theorem.

The proof of $i$ ) and of the last assertion is similar to the proof of Theorem 5.1 and Theorem 5.2. 


\subsection{A Borel-Cantelli property}

A Borel-Cantelli property in dynamical systems has been shown by Philipp [18] for systems related to number theory, by Kleinbock and Margulis [14] for flows on homogeneous spaces and by Chernov and Kleinbock for Gibbs measures [4].

In [6], we have also given a Borel-Cantelli property for a class of positive random variables under the action of $\tau$. As a consequence of Theorem 1.1, we can extend here the corresponding statement (Th. 8.2 of [6] to which we refer for examples and details).

Theorem 6.2. Let us assume (19). Let $\nu$ be the unique P-invariant probability and $\left(f_{n}\right)_{n \geq 0}$ be a sequence of Borel positive functions satisfying

$$
\sum_{n \geq 0} \nu\left(f_{n}\right)=+\infty \quad \text { and } \quad \sup _{n \geq 0}\left\|f_{n}\right\|_{\infty}<+\infty .
$$

If $\sum_{n \geq 0} \sup _{p \geq 0} \tilde{v}\left(f_{p}, n\right)<+\infty$, then the sequence $\left(F_{n}\right)_{n \geq 0}$ defined by

$$
F_{n}=\frac{\sum_{k=0}^{n} f_{k} \circ \tau^{k}}{\sum_{k=0}^{n} \nu\left(f_{k}\right)}, n \geq 0
$$

converges $\nu$-a.e. to 1.

Proof. We show that there exists a positive real $C_{1}$ such that

$$
\int_{E}\left(F_{n}-1\right)^{2} \mathrm{~d} \nu \leq \frac{C_{1}}{\sum_{k=0}^{n} \nu\left(f_{k}\right)}
$$

The theorem will then follow from a classical lemma ( $c f$. [6] for a proof).

Assume $\sum_{n \geq 0} \sup _{k \geq 0} \tilde{v}\left(f_{k}, n\right)<+\infty$. Choose a function $\varphi$ from $\mathbb{N}$ to $] 0,+\infty[$ such that

$$
\sum_{n \geq 0} \varphi(n)=+\infty \text { and } \sum_{n \geq 0}\left(\sup _{k \geq 0}\left|f_{k}\right|_{\varphi, n}\right) \leq \sum_{j \geq 0} \varphi(j) \sum_{n \geq j} \sup _{p \geq 0} \tilde{v}\left(f_{p}, n\right)<+\infty
$$

Setting $g_{k}=f_{k}-\nu\left(f_{k}\right), \forall k \geq 0$, we have:

$$
\begin{aligned}
\left(\sum_{k=0}^{n} \nu\left(f_{k}\right)\right)^{2} \int_{E}\left(F_{n}-1\right)^{2} \mathrm{~d} \nu & \leq 2 \sum_{0 \leq k \leq \ell \leq n} \int_{E} g_{k} \circ \tau^{k} g_{\ell} \circ \tau^{\ell} \mathrm{d} \nu \\
& \leq 2 \sum_{0 \leq k \leq \ell \leq n} \int_{E} g_{\ell} P^{\ell-k} g_{k} \mathrm{~d} \nu \\
& \leq 4 \sum_{0 \leq k \leq \ell \leq n}\left\|P^{\ell-k} g_{k}\right\|_{\infty} \nu\left(f_{\ell}\right)
\end{aligned}
$$

By Lemma 4.2, there is $A>0$ such that:

$$
\forall \ell \geq k \geq 0,\left\|P^{\ell-k} g_{k}\right\|_{\infty} \leq A\left(\left|P^{\ell-k} g_{k}\right|_{\varphi}+\left\|P^{\ell-k} g_{k}\right\|_{1}\right) .
$$


From Theorem 1.1 applied to the triplet $\left(\tilde{B}_{\varphi},\|\|_{1},\left(||_{n}\right)_{n \geq 0}\right)$, we know that, for all $n, k \geq 0$,

$$
\left|P^{n} g_{k}\right|_{\varphi}+\left\|P^{n} g_{k}\right\|_{1} \leq \sum_{p \geq 0}\left(\gamma^{* p} * \beta\right)(n),
$$

with

$$
\beta(n)= \begin{cases}C \sup _{\ell \geq 0}\left|g_{\ell}\right|_{\varphi, n}+C \sum_{j=n-q+1}^{n} a(j-1) \sup _{\ell \geq 0}\left\|g_{\ell}\right\|_{1}, & n \geq q+1 \\ C \sup _{\ell \geq 0}\left|g_{\ell}\right|_{\varphi, n}+C \sum_{j=1}^{n} a(j-1) \sup _{\ell \geq 0}\left\|g_{\ell}\right\|_{1}, & 0 \leq n \leq q \\ 0, & n<0\end{cases}
$$

This implies that there exists $B>0$ such that

$$
\begin{aligned}
\sum_{n \geq 0} \sup _{k \geq 0}\left\|P^{n} g_{k}\right\|_{\infty} & \leq A \sum_{n \geq 0} \sup _{k \geq 0}\left(\left|P^{n} g_{k}\right|_{\varphi}+\left\|P^{n} g_{k}\right\|_{1}\right) \\
& \leq A B\left(\sum_{n \geq 0} \sup _{k \geq 0}\left|g_{k}\right|_{\varphi, n}+\sup _{k \geq 0}\left\|g_{k}\right\|_{1}\right)<+\infty
\end{aligned}
$$

Clearly the result follows.

\subsection{Gibbs measures}

A class of examples is given by subshifts of finite type with a Gibbs measure $\nu$ associated to a regular function. We recall briefly this situation for the full shift. Let $E$ be the product space $E=I^{\mathbb{N}}$, where $I=\left(a_{0}, \cdots, a_{q-1}\right)$ is a finite alphabet with $q \geq 2$, on which operates the shift $\tau: x=\left(x_{0}, x_{1}, \cdots\right) \rightarrow \tau x=\left(x_{1}, x_{2}, \cdots\right)$.

A distance on $E$ is defined by $d(x, y)=1$, if $x_{0} \neq y_{0}$, and $d(x, y)=q^{-(N+1)}$, where $N=\sup \left\{k \in \mathbb{N}: x_{i}=\right.$ $\left.y_{i}, 0 \leq i \leq k\right\}$, otherwise. For the sequence $\left(\eta_{n}\right)$ of $(2.1)$, we take $\eta_{n}=q^{-n}$, for $n \geq 0$.

The family of transformations $S$ is here in bijection with the alphabet $I$ : the transformation $x \rightarrow s x$ is defined by $x=\left(x_{0} x_{1} \ldots\right) \rightarrow s x=\left(s x_{0} x_{1} \ldots\right)$.

Let $u$ be a continuous function on $E$, with strictly positive values, satisfying the condition

$$
\sum_{n \geq 0} \sum_{k \geq n} v(\ln u, k) \leq+\infty
$$

and $P_{u}$ be the operator defined by

$$
P_{u} f(x)=\sum_{s \in S} u(s x) f(s x)
$$

Let $\rho$ be the biggest proper value of $P_{u}$ and $h$ the corresponding proper function ( $c f$. Lem. 3.4). We set

$$
w=\rho^{-1} \frac{h}{h \circ \tau} u \text {. }
$$

This fonction $w$ satisfies the regularity condition: $\sum_{n \geq 0} v(\ln w, n) \leq+\infty$.

Let $P$ be the relativised Markovian operator defined by:

$$
\operatorname{Pf}(x)=\sum_{s \in S} w(s x) f(s x)
$$

The unique $P$-invariant probability measure $\nu$ on the Borel sets of $E$ (Gibbs measure associated to the function $u$ ) is $\tau$-invariant and we have:

$$
\int_{E} \operatorname{Pf} g \mathrm{~d} \nu=\int_{E} f g \circ \tau \mathrm{d} \nu, \forall f, g \in L^{2}(E, \nu) .
$$


The previous results can be applied to the corresponding dynamical system and a $\Phi$-mixing property can be shown as a consequence of Theorem 1.1.

One can ask if Theorem 1.1 can be applied to non uniformly expanding examples. In the last section, we give an example to show how to adapt the arguments in the case of a non uniformly expanding transformation.

\section{Non Uniformly hyperbolic MAPs: An EXAMPle}

We consider the interval $E=[0,1[$, equipped with the Borel $\sigma$-algebra $\mathcal{B}(E)$ and the Lebesgue measure $m$. For a real $x$, we denote by $[x]$ and $\{x\}$ respectively the integral part and fractional part of $x$.

Let $\alpha \in[0,1[$. We consider the transformation $\tau$ of $E$ defined by:

$$
\tau(x)=\left\{\frac{x}{\left(1-x^{\alpha}\right)^{1 / \alpha}}\right\} .
$$

The application $\tau$ has 0 as fixed "indifferent" point and is locally expanding at each other point where it is continuous. For examples of the same type with indifferent fixed point, see $[12,17,20]$ (see also two recent papers [23] and [9] for polynomial estimates for the decay of correlations).

We denote by $P$ the dual operator of the operator $T$ of composition by $\tau$ :

$$
\forall f, g \in \mathbb{L}^{2}(m), \int_{0}^{1} f g \circ \tau \mathrm{d} m=\int_{0}^{1} P f g \mathrm{~d} m .
$$

The measure $m$ is $P$-invariant. Setting, for $x \in E$,

$$
u(x)=\left(1-x^{\alpha}\right)^{1+\frac{1}{\alpha}}, t(x)=\frac{x}{\left(1+x^{\alpha}\right)^{1 / \alpha}} \text { and } \forall k \in \mathbb{N}, s_{k}(x)=t(x+k),
$$

we have:

$$
\forall f \in \mathbb{L}^{2}(m), \forall x \in E, \operatorname{Pf}(x)=\sum_{k \in \mathbb{N}} u\left(s_{k}(x)\right) f\left(s_{k}(x)\right) .
$$

For $j \in \mathbb{N}^{*}$, we have $t^{j}(1)=\frac{1}{(1+j)^{1 / \alpha}}$ and the $j$-th-iterate of $t$ is $t^{j}(x)=\frac{x}{\left(1+j x^{\alpha}\right)^{1 / \alpha}}$. For $x \in E$ and $k \in \mathbb{N}$ we set:

$$
\eta_{k}(x)=\frac{1}{(2+j \vee k)^{1+\frac{1}{\alpha}}}, \quad \text { if } x \in\left[t^{j}(1), t^{j-1}(1)[, j \geq 1 .\right.
$$

Lemma 7.1. For $k \in \mathbb{N}$ and $(x, y) \in E^{2}$ such that $|x-y| \leq \eta_{k}(x)$, we have, for all $\ell \in \mathbb{N},\left|s_{\ell}(x)-s_{\ell}(y)\right| \leq$ $\eta_{k+1}\left(s_{\ell}(x)\right)$.

Proof. Let $x \in\left[\frac{1}{(j+1)^{1 / \alpha}}, \frac{1}{j^{1 / \alpha}}\left[\right.\right.$ and $y \in E$ such that $|x-y| \leq \eta_{k}(x)$.

- For $\ell \geq 1$, we have $s_{\ell}(x) \in\left[1 / 2^{\frac{1}{\alpha}}, 1\left[\right.\right.$ and $\eta_{k+1}\left(s_{\ell}(x)\right)=\frac{1}{(k+3)^{1+1 / \alpha}}$.

$$
\begin{aligned}
\left|s_{\ell}(x)-s_{\ell}(y)\right| & \leq \frac{\eta_{k}(x)}{\left(1+\left(\ell+x-\eta_{k}(x)\right)^{\alpha}\right)^{1+\frac{1}{\alpha}}} \\
& \leq\left[\frac{1}{(j \vee k+2)\left(1+\left(\ell+\frac{1}{(j+1)^{1 / \alpha}}-\frac{1}{(j \vee k+2)^{1+1 / \alpha}}\right)^{\alpha}\right)}\right]^{1+1 / \alpha} \\
& \leq\left[\frac{1}{2(j \vee k+2)}\right]^{1+1 / \alpha} \leq \frac{1}{(k+3)^{1+1 / \alpha}} .
\end{aligned}
$$


- For $\ell=0$, we have:

$$
|t(x)-t(y)| \leq\left[\frac{1}{(j \vee k+2)\left(1+\left(\frac{1}{(j+1)^{1 / \alpha}}-\frac{1}{(j \vee k+2)^{1+1 / \alpha}}\right)^{\alpha}\right)}\right]^{1+1 / \alpha} .
$$

Since

$$
(j \vee k+2)\left(\frac{1}{(j+1)^{1 / \alpha}}-\frac{1}{(j \vee k+2)^{1+1 / \alpha}}\right)^{\alpha} \geq \frac{(j \vee k+2)}{j+1}\left(1-\frac{1}{j \vee k+2}\right)^{\alpha} \geq 1 ;
$$

this implies $|t(x)-t(y)| \leq\left[\frac{1}{j \vee k+3}\right]^{1+1 / \alpha}=\eta_{k+1}(t(x))$.

Let $f$ be a Borel function on $E, x \in E$ and $n \in \mathbb{N}$. We set:

$$
\begin{aligned}
\widetilde{\mathcal{V}}(f, x, n) & =\|f(x)-f(\cdot)\|_{L^{\infty}\left(B\left(x, \eta_{n}(x)\right), m\right)}, \\
\widetilde{v}(f, n) & =\int_{0}^{1} \tilde{\mathcal{V}}(f, x, n) \mathrm{d} x .
\end{aligned}
$$

We denote by $V$ the subspace $V=\left\{f \in \mathbb{L}^{1}(E, m): \lim _{n \rightarrow+\infty} \tilde{v}(f, n)=0\right\}$.

Theorem 7.2. The sequence $\left(P^{n} 1\right)_{n \geq 0}$ converges uniformly on compacts of $\left.] 0,1\right]$ to a strictly positive, continuous, $P$-invariant function $h$.

If $f$ is in $V$, the sequence $\left(P^{n} f\right)_{n \geq 0}$ converges in $L^{1}(m)$-norm to $m(f) h$.

Theorem 7.2 will result from the following lemmas.

We set

$$
\begin{aligned}
w(k) & =\sup _{x \in E} \sup _{y \in B\left(x, \eta_{k}(x)\right)} \sup _{n \geq 0}\left|\ln u\left(s_{n}(x)\right)-\ln u\left(s_{n}(y)\right)\right|, \\
z(k) & =\mathrm{e}^{w(k)} w(k) .
\end{aligned}
$$

Lemma 7.3. $\forall k \in \mathbb{N}, w(k) \leq(\alpha+1) k^{-2}$.

Proof. We set $\psi=\ln (u \circ t)$. For all $x \in E$, we have:

$$
\psi(x)=-\left(1+\frac{1}{\alpha}\right) \ln \left(1+x^{\alpha}\right) \text { and } \psi^{\prime}(x)=-(1+\alpha) \frac{1}{x+x^{1-\alpha}} .
$$

Hence, for $k \in \mathbb{N}$ and $x, y \in E$ such that $|x-y| \leq \eta_{k}(x)$, we have

$$
|\psi(x+n)-\psi(y+n)| \leq(1+\alpha) \frac{|x-y|}{(x \wedge y)^{1-\alpha}} \leq(1+\alpha) \frac{\eta_{k}(x)}{\left(x-\eta_{k}(x)\right)^{1-\alpha}} .
$$

The lemma follows then from the inequality:

$$
\sup _{x \in E} \frac{\eta_{k}(x)}{\left(x-\eta_{k}(x)\right)^{1-\alpha}} \leq \frac{1}{k^{2}} .
$$


By the same proof as in Lemma 4.4, we have:

Lemma 7.4. If $f$ is a function on $E$, we have:

$$
\widetilde{v}(P f, k) \leq \mathrm{e}^{z(k)} \widetilde{v}(f, k+1)+z(k)\|f\|_{1}, \forall k \geq 0 .
$$

Lemma 7.5. For all $k \in \mathbb{N}, j \in \mathbb{N}^{*}$ and $f \in \mathcal{L}^{\infty}\left(\left[t^{j}(1), 1[, m)\right.\right.$, we have

$$
\|f\|_{L^{\infty}\left(\left[t^{j}(1), 1[, m)\right.\right.} \leq 2^{-1}(2+j \vee k)^{1+\frac{1}{\alpha}}\left(\tilde{v}(f, k)+\|f\|_{1}\right) .
$$

Proof. Let $y \in\left[t^{j}(1), 1\left[\right.\right.$, we have $\eta_{k}(y) \geq \eta_{k}\left(t^{j}(1)\right)$; so that for $m$-almost all $x \in\left[t^{j}(1), 1[\right.$, we have:

$$
\begin{aligned}
1_{B\left(x, \eta_{k}\left(t^{j}(1)\right)\right)}(y)|f(x)| & \leq 1_{B\left(x, \eta_{k}\left(t^{j}(1)\right)\right)}(y)(|f(x)|-|f(y)|)+|f(y)| \\
& \leq\|f(\cdot)-f(y)\|_{L^{\infty}\left(E, B\left(y, \eta_{k}\left(t^{j}(1)\right)\right), m\right)}+|f(y)| \\
& \leq \tilde{\mathcal{V}}(f, y, k)+|f(y)| .
\end{aligned}
$$

We obtain the result after integration.

Notation. For $f \in V$ and $k \in \mathbb{N}$, we set

$$
\delta(f, k)=\int_{0}^{t^{k+1}(1)}|f(x)| \mathrm{d} x+c \mathrm{e}^{\sum_{\ell \geq 0} z(\ell)} \sum_{j=0}^{k} t^{k-j}(1) \mathrm{e}^{-\sum_{\ell \geq j} z(\ell)} \tilde{v}(f, j),
$$

where $c=\frac{3}{2}(1+1 / \alpha) 3^{1 / \alpha}$.

\section{Lemma 7.6.}

$$
\delta(P f, k) \leq \delta(f, k+1)+c \mathrm{e}^{\sum_{\ell \geq 0} z(\ell)}\left(t^{k+1}(1)+\sum_{j=0}^{k} t^{k-j}(1) z(j)\right)\|f\|_{1} .
$$

Proof. We can assume $f \geq 0$. The following inequalities hold:

$$
\begin{aligned}
\int_{0}^{t^{k+1}(1)} P f(x) \mathrm{d} x & \leq \sum_{n \geq 0} \int_{0}^{t^{k+1}(1)} u(t(x+n)) f(t(x+n)) \mathrm{d} x \\
& =\int_{0}^{t^{k+2}(1)} f(x) \mathrm{d} x+\sum_{n \geq 1} \int_{t(n)}^{t\left(t^{k+1}(1)+n\right)} f(y) \mathrm{d} y \\
& \leq \int_{0}^{t^{k+2}(1)} f(x) \mathrm{d} x+\|f\|_{L^{\infty}([t(1), 1[, m)} \sum_{n \geq 1}\left(t\left(t^{k+1}(1)+n\right)-t(n)\right) \\
& \leq \int_{0}^{t^{k+2}(1)} f(x) \mathrm{d} x+\|f\|_{L^{\infty}([t(1), 1[, m)} t^{k+1}(1) \sum_{n \geq 1} \frac{1}{n^{1+\alpha}} \\
& \leq \int_{0}^{t^{k+2}(1)} f(x) \mathrm{d} x+c t^{k+1}(1)\left(\tilde{v}(f, 0)+\|f\|_{1}\right) ;
\end{aligned}
$$




$$
\begin{aligned}
\sum_{j=0}^{k} t^{k-j}(1) \mathrm{e}^{-\sum_{\ell \geq j} z(\ell)} \tilde{v}(P f, j) & \leq \sum_{j=0}^{k} t^{k-j}(1) \mathrm{e}^{-\sum_{\ell \geq j} z(\ell)}\left(\mathrm{e}^{z(j)} \tilde{v}(f, j+1)+z(j)\|f\|_{1}\right) \\
& \leq \sum_{j=1}^{k+1} t^{k+1-j}(1) \mathrm{e}^{-\sum_{\ell \geq j} z(\ell)} \tilde{v}(f, j)+\sum_{j=0}^{k} t^{k-j}(1) z(j)\|f\|_{1} .
\end{aligned}
$$

The result follows.

Let $g \in V$. We choose a decreasing function $\varphi$ from $\mathbb{N}$ to $] 0,+\infty[$ such that

$$
\sum_{k \geq 0} \varphi(k)=+\infty \text { and } \sum_{k \geq 0} \varphi(k)\left(\tilde{v}(g, k)+\int_{0}^{t^{k}(1)}|g(x)| \mathrm{d} x\right)<+\infty .
$$

We can replace $\varphi(n)$ with $\varphi(n) \wedge n^{-1}$, and assume that $\varphi(n) \leq n^{-1}$, so that the following series converges:

$$
\sum_{k \geq 0} \varphi(k) \sum_{j \geq k} w(j) \text { and } \sum_{k \geq 0} \varphi(k) \delta(g, k)
$$

We introduce the notations:

$$
\begin{aligned}
\forall n \in \mathbb{N},|f|_{\varphi, n} & =\sum_{k \geq 0} \varphi(k)(\tilde{v}(f, k+n)+\delta(f, k+n)), \\
|f|_{\varphi} & =|f|_{\varphi, 0}=\sum_{k \geq 0} \varphi(k)(\tilde{v}(f, k)+\delta(f, k)) .
\end{aligned}
$$

Let $\tilde{B}_{\varphi}$ be the subspace of $L^{1}(E, \nu)$ defined by $\tilde{B}_{\varphi}=\left\{f \in L^{1}(E, \nu):|f|_{\varphi}<+\infty\right\}$. The following three lemmas show that $\left(P, \tilde{B}_{\varphi},\|\|_{1},\left(||_{\varphi, n}\right)_{n \geq 0}\right)$ satisfies the hypotheses of Theorem 1.1.

Lemma 7.7. The unit ball $\mathcal{B}_{1}=\left\{f \in \tilde{B}_{\varphi}:|f|_{\varphi}+\|f\|_{1} \leq 1\right\}$ is compact in $\left(\tilde{B}_{\varphi},\|\|_{1}\right)$.

Proof. We adapt the proof of Lemma 4.4 to the non uniformly hyperbolic example.

For each integer $k \geq 1$, consider a finite open covering of the compact set $K_{k}=\left[t^{k}(1), 1\right]$

$$
K_{k}=\bigcup_{1 \leq j \leq p_{k}} B\left(x_{j, k}, \eta_{k}\left(x_{j, k}\right)\right) .
$$

Let $\left(\psi_{j, k}\right)_{1 \leq j \leq p_{k}}$ be a partition of unity subordinate to this covering (i.e. the functions $\psi_{j, k}, k \geq 0,1 \leq j \leq p_{k}$, are non-negative continuous and satisfy

$$
\forall x \in K_{k}, \sum_{j=1}^{k} \psi_{j, k}(x)=1 \text { and } \forall x \notin B\left(x_{j, k}, \eta_{k}\left(x_{j, k}\right)\right), \psi_{j, k}(x)=0 .
$$


Let $\left(f_{n}\right)_{n \geq 0}$ be a sequence of functions in $\mathcal{B}_{1}$. By Lemma 7.5, we may assume that these functions are bounded on $K_{k}$ by $2^{-1}(1+k)^{1+\frac{1}{\alpha}}\left((\varphi(0))^{-1}+1\right)$. For all integers $k, n$ and $p$, we have:

$$
\begin{aligned}
\left\|f_{n+p}-f_{n}\right\|_{L^{1}\left(K_{k}, m\right)}= & \sum_{j=1}^{p_{k}} \int_{K_{k}} \psi_{j, k}\left|f_{n+p}-f_{n}\right| \mathrm{d} m \\
\leq & \sum_{j=1}^{p_{k}}\left(\int_{K_{k}} \psi_{j, k}\left|f_{n+p}-f_{n+p}\left(x_{j, k}\right)\right| \mathrm{d} m+\int_{K_{k}} \psi_{j, k}\left|f_{n}-f_{n}\left(x_{j, k}\right)\right| \mathrm{d} m\right. \\
& \left.+\int_{K_{k}} \psi_{j, k} \mathrm{~d} m\left|f_{n+p}\left(x_{j, k}\right)-f_{n}\left(x_{j, k}\right)\right|\right) \\
\leq & \sum_{j=1}^{p_{k}}\left(\int_{K_{k}} \psi_{j, k} \widetilde{\mathcal{V}}\left(f_{n+p}, \cdot, k\right) \mathrm{d} m+\int_{K_{k}} \psi_{j, k} \widetilde{\mathcal{V}}\left(f_{n}, \cdot, k\right) \mathrm{d} m\right. \\
& \left.+\int_{K_{k}} \psi_{j, k} \mathrm{~d} m\left|f_{n+p}\left(x_{j, k}\right)-f_{n}\left(x_{j, k}\right)\right|\right) \\
\leq & \widetilde{v}\left(f_{n+p}, k\right)+\widetilde{v}\left(f_{n}, k\right)+\sum_{j=1}^{p_{k}} \int_{K_{k}} \psi_{j, k} \mathrm{~d} m\left|f_{n+p}\left(x_{j, k}\right)-f_{n}\left(x_{j, k}\right)\right| \\
\leq & 2\left(\sum_{j=0}^{k} \varphi(j)\right)^{-1}+\sum_{j=1}^{p_{k}} \int_{K_{k}} \psi_{j, k} \mathrm{~d} m\left|f_{n+p}\left(x_{j, k}\right)-f_{n}\left(x_{j, k}\right)\right| .
\end{aligned}
$$

As

$$
\left\|f_{n+p}-f_{n}\right\|_{L^{1}\left(K_{k}^{c}, m\right)} \leq \int_{0}^{t^{k}(1)}\left|f_{n+p}\right| \mathrm{d} m+\int_{0}^{t^{k}(1)}\left|f_{n}\right| \mathrm{d} m \leq 2\left(\sum_{j=0}^{k} \varphi(j)\right)^{-1}
$$

we have

$$
\left\|f_{n+p}-f_{n}\right\|_{1} \leq 4\left(\sum_{j=0}^{k} \varphi(j)\right)^{-1}+\sum_{j=1}^{p_{k}} \int_{K_{k}} \psi_{j, k} \mathrm{~d} m\left|f_{n+p}\left(x_{j, k}\right)-f_{n}\left(x_{j, k}\right)\right| .
$$

If $(\sigma(n))_{n \geq 0}$ is a strictly increasing sequence of integers such that the real sequences $\left(f_{\sigma(n)}\left(x_{j, k}\right)\right)_{n \geq 0}$ converge, for every $k \geq 0$ and $j \in\left[1, p_{k}\right]$, then the sequence of functions $\left(f_{\sigma(n)}\right)_{n \geq 0}$ is a Cauchy sequence in $L^{1}(E, m)$.

This shows that the set $\mathcal{B}_{1}$ is relatively compact in $L^{1}(E, m)$. It remains to show that $\mathcal{B}_{1}$ is closed.

Let $\left(f_{n}\right)_{n>0}$ be a sequence of functions in $\mathcal{B}_{1}$ which converges in $L^{1}(E, m)$ to a function $f$. Taking if necessary a subsequence, we can assume that the sequence $\left(f_{n}\right)_{n \geq 0}$ converges $m$-a.e. to $f$. From Egorov's theorem, there exists an increasing sequence $\left(E_{p}\right)_{p \geq 0}$ of the Borel sets of $E$ such that

$$
\lim _{p \rightarrow+\infty} m\left(E_{p}\right)=1 \quad \text { and } \quad \forall p \geq 0, \lim _{n \rightarrow+\infty}\left\|f_{n}-f\right\|_{L^{\infty}\left(E_{p}, m\right)}=0 .
$$

For all integers $k, p \geq 0$, we have then:

$$
\begin{aligned}
& \int_{E_{p}}\|f(x)-f(\cdot)\|_{L^{\infty}\left(B\left(x, \eta_{k}(x)\right) \cap E_{p}, m\right)} \mathrm{d} x=\lim _{n \rightarrow+\infty} \int_{E_{p}}\left\|f_{n}(x)-f_{n}(\cdot)\right\|_{L^{\infty}\left(B\left(x, \eta_{k}(x)\right) \cap E_{p}, m\right)} \mathrm{d} x \\
& \leq \limsup _{n \rightarrow+\infty} \int_{E_{p}}\left\|f_{n}(x)-f_{n}(\cdot)\right\|_{L^{\infty}\left(B\left(x, \eta_{k}(x)\right), m\right)} \mathrm{d} x \text {. }
\end{aligned}
$$

From this, we easily deduce that $|f|_{\varphi} \leq \sup _{n \geq 0}\left|f_{n}\right|_{\varphi} \leq 1$. 
From Lemmas 7.4 and 7.6, it follows:

Lemma 7.8. We have $|P f|_{\varphi, k} \leq \mathrm{e}^{z(k)}|f|_{\varphi, k+1}+a_{k}\|f\|_{1}$, where

$$
a_{k}=\sum_{j \geq 0} \varphi(j)\left(z(k+j)+C \mathrm{e}^{\sum_{\ell \geq 0} z(\ell)}\left(t^{k+j}(1)+\sum_{\ell=0}^{k+j} t^{k+j-\ell}(1) z(\ell)\right) .\right.
$$

Lemma 7.9. For all $f \in \tilde{B}_{\varphi}, \lim _{n \rightarrow+\infty}|f|_{\varphi, n}=0$.

Proof. From the definition (25), we have for some constant $C: \delta(f, k) \leq \int_{0}^{t^{k+1}(1)}|f(x)| \mathrm{d} x+C \sum_{j=0}^{k} t^{k-j}(1) \tilde{v}(f, j)$, so that it is enough to show that $\lim _{n \rightarrow+\infty} \sum_{k \geq 0} \varphi(k) u(n+k)=0$, where $u(n)=\sum_{j=0}^{n}(n-j+1)^{-1 / \alpha} \widetilde{v}(f, j)$.

We have:

$$
\begin{aligned}
u(k+n) & \leq \sum_{j=0}^{\left[\frac{n+k}{2}\right]} \widetilde{v}(f, j)\left(\left[\frac{n+k}{2}\right]+1\right)^{-1 / \alpha}+\widetilde{v}\left(f,\left[\frac{n+k}{2}\right]\right) \sum_{k \geq 1} k^{-1 / \alpha} \\
& \leq \sup _{p \geq\left[\frac{n}{2}\right]}\left(p^{-1} \sum_{j=0}^{p} \widetilde{v}(f, j)\right)\left(\left[\frac{n+k}{2}\right]+1\right)^{1-(1 / \alpha)}+\widetilde{v}\left(f,\left[\frac{n+k}{2}\right]+1\right) \sum_{k \geq 1} k^{-1 / \alpha} .
\end{aligned}
$$

The result follows then from the inequality:

$$
\begin{aligned}
\sum_{k \geq 0} \varphi(k) \widetilde{v}\left(f,\left[\frac{n+k}{2}\right]\right) & =\sum_{k \geq 0} \varphi(2 k) \widetilde{v}\left(f,\left[\frac{n}{2}\right]+k\right)+\sum_{k \geq 0} \varphi(2 k+1) \widetilde{v}\left(f,\left[\frac{n+1}{2}\right]+k\right) \\
& \leq 2 \sum_{k \geq 0} \varphi(k) \widetilde{v}\left(f,\left[\frac{n}{2}\right]+k\right)
\end{aligned}
$$

and from the analogous inequality for $\sum_{k \geq 0} \varphi(k)\left(\left[\frac{n+k}{2}\right]+1\right)^{1-(1 / \alpha)}$.

Now we know that $\left(P, \tilde{B}_{\varphi},\|\|_{1},\left(||_{\varphi, n}\right)_{n \geq 0}\right)$ satisfies the hypotheses of Theorem 1.1. From this theorem, it follows that:

1) the subspace $W_{1}$ of $\tilde{B}_{\varphi}$ generated by the eigenvectors of $P$ corresponding to eigenvalues of modulus 1 is finite dimensional;

2) we have $B=W_{1} \oplus W_{2}$, where $W_{2}=\left\{f \in B: \lim _{n \rightarrow+\infty}\left\|P^{n} f\right\|_{1}=0\right\}$;

3 ) if $\lambda$ is a complex number of modulus 1 and $f$ is in $B$, the sequence

$$
\left(\frac{f+\lambda^{-1} P f+\cdot+\lambda^{-(n-1)} P^{n-1} f}{n}\right)_{n \geq 1}
$$

converges in $L^{1}(m)$-norm either to zero or to a $\lambda$-eigenvector of $P$.

Lemma 7.10. The sequence of continuous functions $\left(P^{n} 1\right)_{n \geq 0}$ converges uniformly on compact sets of $\left.] 0,1\right]$ to a continuous, strictly positive and $P$-invariant function $h$.

Proof. We know that the sequence $\left(\frac{1+P 1+\cdot+P^{n-1} 1}{n}\right)_{n \geq 1}$ converges in $L^{1}(m)$-norm to a non negative $P$-invariant function. 
We know also (cf. (17), proof of Lem. 3.4), that if $f$ is a non negative function and $k \in \mathbb{N}$, we have: $v(\ln P f, k) \leq v(\ln f, k+1)+w(k)$. It follows that, for $n \in \mathbb{N}, v\left(\ln P^{n} 1, k\right) \leq \sum_{j \geq k} w(j)$ and the family of functions $\left\{P^{n} 1: n \geq 0\right\}$ is equicontinous. From Lemma 7.5, this family is also bounded on the compact subsets of $] 0,1]$. By Ascoli's theorem $\left\{P^{n} 1: n \geq 0\right\}$ is therefore relatively compact for the uniform convergence on compact sets of $] 0,1]$.

These properties imply that the sequence of continuous functions $\left(P^{n} 1\right)_{n \geq 0}$ converges uniformly on compact sets of $] 0,1]$ to a continuous, non negative, $P$-invariant function $h$ satisfying $\int_{0}^{1} h(x) \mathrm{d} x=1$.

If $h\left(x_{0}\right)=0$ for some $\left.\left.x_{0} \in\right] 0,1\right]$ then $h\left(s_{j_{1}} \cdots s_{j_{p}}\left(x_{0}\right)\right)=0$ for all $p \geq 1$ and all $\left(j_{1}, \ldots, j_{p}\right) \in \mathbb{N}^{p}$. From the density of the subset $\left\{s_{j_{1}} \cdots s_{j_{p}}\left(x_{0}\right): p \geq 1,\left(j_{1}, \ldots, j_{p}\right) \in \mathbb{N}^{p}\right\}$ in $\left.] 0,1\right]$, it follows that $h$ is the nullfunction on ]0,1] contradicting the fact that $\int_{0}^{1} h(x) \mathrm{d} x=1$.

To conclude the proof of Theorem 7.2, we have to show that the dimension of $W_{1}$ is one.

Lemma 7.11. $W_{1}=\mathbb{C} h$

Proof. 1) Let $g \in \tilde{B}_{\varphi}$ be a real $P$-invariant function. Due to the $P$-invariance of the measure $m$, the functions $g^{+}$and $g^{-}$are also $P$-invariant so that we can assume that $g$ is non negative.

For all positive reals $a$, the function $g \wedge a h$ is also $P$-invariant and $g_{a}=\frac{g \wedge a h}{h}$ is ${ }^{h} P$-invariant, where ${ }^{h} P$ is the relativised operator defined by ${ }^{h} P=\frac{1}{h} P(h f)$. We have, for $r \in \mathbb{N}^{*}$,

$$
\begin{aligned}
\int_{0}^{1} \int_{0}^{1}\left(g_{a}(x)-g_{a}(u)\right)^{2 h} P^{r}(u, \mathrm{~d} x) h(u) \mathrm{d} u & =\int_{E}\left({ }^{h} P^{r}\left(g_{a}^{2}\right)+g_{a}^{2}-2 g_{a}{ }^{h} P^{r} g_{a}\right) h \mathrm{~d} m \\
& =\int_{E}\left({ }^{h} P^{r}\left(g_{a}^{2}\right)-g_{a}^{2}\right) h \mathrm{~d} m=0 .
\end{aligned}
$$

It follows that, for all $r \geq 1$ and all $\left(j_{1}, \ldots, j_{r}\right) \in \mathbb{N}^{p}, g_{a}\left(s_{j_{1}} \cdots s_{j_{r}}(u)\right)=g_{a}(u)$, for $m$-almost all $u \in E$.

As $g$ belongs to $\tilde{B}_{\varphi}$, the function $C(x)=\sum_{k \geq 0} \varphi(k)\|g(x)-g\|_{L^{\infty}\left(B\left(x, \eta_{k}(x)\right)\right.}$ is $m$-integrable and therefore $m$-almost everywhere finite. For all $n \geq 0$ and $x \in] 0,1]$ such that $C(x)<+\infty$, we have:

$$
\|g(x)-g\|_{L^{\infty}\left(B\left(x, \eta_{n}(x)\right)\right.} \leq \frac{C(x)}{\sum_{k=0}^{n} \varphi(k)} \underset{n \rightarrow+\infty}{\longrightarrow} 0 .
$$

Let $x \in\{C<+\infty\}$. Choose a sequence of natural integers $\left(j_{k}\right)_{k \geq 1}$ such that, for every $\left.\left.u \in\right] 0,1\right], s_{j_{1}} \cdots s_{j_{n}}(u) \rightarrow$ $x$. For $m$-almost all $u \in] 0,1]$, we have $g\left(s_{j_{1}} \cdots s_{j_{n}}(u)\right) \rightarrow g(x)$ and therefore $g_{a}\left(s_{j_{1}} \cdots s_{j_{n}}(u)\right)=g_{a}(u) \rightarrow g_{a}(x)$, so that $g_{a}$ is $m$-a.e. constant.

For every $a>0, g \wedge a h$ belongs to $\mathbb{C} h$ and therefore $g$ belongs to $\mathbb{C} h$.

2) Let $g \in \tilde{B}_{\varphi}$ such that $P g=\lambda g$, for an eigenvalue $\lambda$ of modulus 1. The function $|g|$ is $P$-invariant. From 1) it follows that $g$ takes its values in $\mathbb{C}^{*}$ and the function $f=\frac{g}{|g|}$ is a ${ }^{h} P$-eigenfunction corresponding the eigenvalue $\lambda$. As before we have, for $r \geq 1$,

$$
\int_{0}^{1} \int_{0}^{1}\left|f(x)-\lambda^{r} f(u)\right|^{2}{ }^{h} P^{r}(u, \mathrm{~d} x) h(u) \mathrm{d} u=0
$$

and for $m$-almost all $u \in E, f\left(s_{j_{1}} \cdots s_{j_{r}}(u)\right)=\lambda^{r} f(u)$, for all $r \geq 1$ and all $\left(j_{1}, \ldots, j_{r}\right) \in \mathbb{N}^{p}$. The same argument as before shows that $f$ is $m$-a.e. constant. 
Remark. Let $f$ be $\in W_{2}$ such that $\tilde{v}(f, n)+\int_{0}^{t^{n}(1)}|f(x)| \mathrm{d} x=O\left(n^{-a}\right)$, for some $a>0$. Using an inequality similar to the inequality (1) of Theorem 1.1 and the fact that, for all $a>0$, there exists $C>0$ such that:

$$
\sum_{j=0}^{n}(j+1)^{-a}(n+1-j)^{-1 / \alpha} \leq C(n+1)^{-(a \wedge(1 / \alpha))},
$$

we get, for every $\varepsilon>0$ :

$$
\left\|P^{n} f\right\|_{1}=o\left(n^{-\left(a \wedge 2 \wedge \frac{1}{\alpha}\right)+\varepsilon}\right) .
$$

\section{REFERENCES}

[1] V. Baladi, Positive Transfer Operators and Decay of Correlations. World Scientific, Adv. Ser. Nonlinear Dynam. 16 (2000).

[2] R. Bowen, Equilibrium states and the ergodic theory of Anosov Diffeomorphisms. Springer-Verlag, Lectures Notes 470 (1975).

[3] B.M. Brown, Martingale central limit theorem. Ann. Math. Statist. 42 (1971) 59-66.

[4] N. Chernov and D. Kleinbock, Dynamical Borel-Cantelli lemmas for Gibbs measures. Isreal J. Math. 122 (2001) 1-27.

[5] J.-P. Conze and A. Raugi, Fonctions harmoniques pour un opérateur de transition et applications. Bull. Soc. Math. France 118 (1990) 273-310.

[6] J.-P. Conze and A. Raugi, Convergence des potentiels pour un opérateur de transfert, applications aux systèmes dynamiques et aux chaînes de Markov. Séminaires de Rennes (1998) 52.

[7] M.I. Gordin, On the central limit theorem for stationary processes. Dokl. Akad. Nauk SSSR, Soviet Math. Dokl. 10 (1969) 1174-1176.

[8] M.I. Gordin and B.A. Lifvsic, Central limit theorem for stationary Markov processes. Dokl. Akad. Nauk SSSR 239 (1978) 766-767.

[9] S. Gouëzel, Sharp polynomial estimates for the decay of correlations. Preprint (2002).

[10] P. Hall and C.C. Heyde, Martingale limit theory and its applications. Academic Press, New York (1980).

[11] H. Hennion and L. Hervé, Limit theorem for Markov Chains and Stochastic Properties of Dynamical Systems by Quasicompactness. Springer-Verlag, Lectures Notes 1766 (2001).

[12] H. Hu, Decay of correlations for piecwise smooth maps with indifferent fixed points. Preprint.

[13] C. Jan, Vitesse de convergence dans le TCL pour certaines chaînes de Markov et certains systèmes dynamiques, Preprint. Université de Rennes 1 (2000).

[14] D.Y. Kleinbock and G.A. Margulis, Logarithm laws for flows on homogeneous spaces. Invent. Math. 138 (1999) $451-494$.

[15] A. Kondah, V. Maume and B. Schmitt, Vitesse de convergence vers l'état d'équilibre pour des dynamiques markoviennes non höldériennes. Ann. Inst. H. Poincaré 33 (1997) 675-695.

[16] C. Liverani, Decay of correlations. Ann. Math. 142 (1995) 239-301.

[17] C. Liverani, B. Saussol and S. Vaienti, A probabilistic approach to intermittency. Ergodic Theory Dynam. Systems 19 (1999) 671-685.

[18] W. Philipp, Some metrical theorems in number theory. Pacific J. Math. 20 (1967) 109-127.

[19] M. Pollicott, Rates of mixing for potentials of summable variation. Trans. Amer. Math. Soc. 352 (2000) 843-853.

[20] M. Pollicott and M. Yuri, Statistical properties of maps with indifferent periodic points. Comm. Math. Phys. 217 (2001) 503-520.

[21] A. Raugi, Théorie spectrale d'un opérateur de transition sur un espace métrique compact. Ann. Inst. H. Poincaré 28 (1992) 281-309.

[22] E. Rio, Sur le théorème de Berry-Esseen pour les suites faiblement dépendantes. J. Probab. Theory Related Fields 104 (1996) 255-282.

[23] O. Sarig, Subexponential decay of decorrelation. Preprint (2001).

[24] Ya.G. Sinai, Gibbs measures in ergodic theory. Russian Math. Surveys 166 (1972) 21-64.

[25] P. Walters, Invariant measures and equilibrium states for some mappings which expand distances. Trans. Amer. Math. Soc. 236 (1978) 121-153.

[26] L.-S. Young, Recurrence times and rates of mixing. Israel J. Math. 110 (1999) 153-188. 